\title{
Asymptotic Models for Studying Kinetics of Formation of Compact Objects with Strong Internal Bonds
}

\author{
Emil Edipovich Lin \\ Russian Federal Nuclear Center-All-Russia Research Institute of Experimental Physics, Sarov, Russia \\ Email: postmaster@ifv.vniief.ru, e.e.lin@ifv.vniief.ru
}

Received 30 March 2014; revised 24 April 2014; accepted 23 May 2014

Copyright (C) 2014 by author and Scientific Research Publishing Inc. This work is licensed under the Creative Commons Attribution International License (CC BY). http://creativecommons.org/licenses/by/4.0/ c) (i) Open Access

\section{Abstract}

An asymptotic method has been developed for investigation of kinetics of formation of compact objects with strong internal bonds. The method is based on the uncertainty relation for a coordinate and a momentum in space of sizes of objects (clusters) with strongly pronounced collective quantum properties resulted from exchange interactions of various physical nature determined by spatial scales of the processes under consideration. The proposed phenomenological approach has been developed by analogy with the all-known ideas about coherent states of quantum mechanical oscillator systems for which a product of coordinate and momentum uncertainties (dispersions) accepts the value, which is minimally possible within uncertainty relations. With such an approach the leading processes are oscillations of components that make up objects, mainly: collective nucleon oscillations in a nucleus and phonon excitations in a mesostructure crystal lattice. This allows us to consider formation and growth of subatomic and mesoscopic objects in the context of a single formalism. The proposed models adequately describe characteristics of formation processes of nuclear matter clusters as well as mesoscopic crystals having covalent and quasi-covalent bonds between atoms.

\section{Keywords}

Compact Objects, Formation Kinetics, Clusters, Quantum Properties, Spatial Scales, Nuclear Matter, Mesoscopic Crystals

\section{Introduction}

In works [1]-[6] various physical objects and their environment are described. They are of interest not only in 
terms of their individual properties determined by their structure and sizes but also in terms of determining a spatial boundary between macro- and microcosm (macrophysics and microphysics). Object sizes "intermediate" between atoms and solid bodies in mesoscopic crystal structures (in which behaviors conform to the rules of both quantum mechanics and classical physics) can reach considerable values, at which the lower complexity limit of a macroscopic crystal is reached. Fundamental considerations and assessments of quantum representation feasibility show that the "coordinate-momentum" uncertainty relation can fit for bodies with the sizes about $10^{-7} \mathrm{~m}$, i.e., at a level of nanometer scales [2]. Currently more and more papers deal with investigations of formation, evolution and properties of nanostructures and nanostructure-based materials. These investigations cover processes of various physical and chemical nature proceeding in a mesoscopic region of spatial scales up to $10^{-10}-10^{-4} \mathrm{~m}$ : from formation of atomic clusters on a molecular level and nanocrystal synthesis to global changes of a macroscopic continuum state [7]-[13]. Mechanical behavior of nanostructured objects is described using molecular dynamics methods and using statistic models allowing for both thermodynamic and kinetic aspects of problems under consideration. These problems cover incompleteness of classical description of process running in mesoscopic scales, necessity of developing new models including phenomenological ones that take into account formation of quantum systems and determine growth regularities and nanostructure consolidation, and determination of phase transformation size dependencies, in particular, ascertainment of the effect of polymorphic transformations on synergy of crystal structures.

In this connection it is important in principle to determine general features of dynamics of object growth both in microcosm and in "mesocosm". With this purpose this paper investigates kinetics of formation of various in physical nature compact objects having strong internal bonds and considerably different spatial scales: nuclear matter clusters [14] and nanostructured mesoscopic structures with covalent and quasi-covalent bonds between atoms in a crystal lattice [15]. A typical feature of these objects is the existence of purely quantum effect of exchange interaction. Virtual meson exchange occurs between nucleons; electron exchange occurs in crystal structures having covalent bonds. The effect of exchange interaction allows these objects to be viewed as compact clusters with strongly pronounced collective quantum properties ("quantum" clusters). In case of nuclei these quantum properties are connected with strong interactions and manifest themselves in the existence of oscillatory and rotator shells. In mesocrystals quasi-particles (phonons) are excited.

The above creates the necessary precondition for investigating kinetics of object formation from a common point of view. We will consider closed stochastic systems of compact quantum clusters randomly interacting with each other. In accordance with standard ideas of physical kinetics the irreversible aggregation of objects is described using the concept of distribution density wave $\varphi(a, t)$ in the space of cluster sizes $a$, propagating in time $t$ in direction of cluster size increase. It is noted in Ref. [16] that the behavior of a wave packet of any physical nature is subject to a universal ratio (resulting from Fourier theorem) for half-width of a wave packet and half-width of a spectral line $\Delta a \cdot \Delta k \geq 1 / 4 \pi$, where $k$ is a wave number. This results in the uncertainty relation for coordinate and momentum in the space of cluster sizes. For quantum-mechanical systems being in a coherent state (harmonic oscillations), the above product takes the minimum value, the uncertainty relation is fulfilled as an accurate equality, and a process trajectory in a phase space is classical [17]. This means that it is possible to consider relatively large objects, the sizes of which exceed values corresponding to the lower limit of complexity for a macroscopic solid. Thus, the proposed phenomenological approach describes a limiting asymptotic stage of cluster growth process. Under this approach "the leading" processes appear to be oscillations of components that compose objects, for example collective oscillations of nucleons in a nucleus or phonon excitations of mesostructure crystal lattice.

It should be noted that the proposed asymptotic method in no way can replace the developed fundamental methods of investigations and physical models of microcosm and mesocosm dynamics described in the above Refs. [1]-[10] and represented in [18]-[21]. On the other hand it is well known that "when interacting with outer space, physical objects never discover their complete internal complexity potential. Hence incomplete or even phenomenological description of physical phenomena or physical objects sometimes responses better to the main point, and to the understanding of what is going on" [2].

For example, when solving kinetic equations, the fitting parameters determined using experiments are introduced, as a rule. When planning experiments with new or weakly studied objects, there appears necessity to perform preliminary evaluations of final dimensional and timing characteristics of the investigated processes, i.e., to determine dependence between typical object sizes and the time of their growth. In this respect, the proposed asymptotic models can be useful when first meeting this or that problem, evaluating it or analyzing pri- 
mary experimental data. For example, when studying cluster radioactivity described in [22], this method made it possible to determine the spectrum of the most probable mass numbers of relatively light nucleon clusters formed inside heavy nuclei [14] [23]. This method also makes it possible to evaluate both average and maximum mass numbers of super-heavy nuclei and formed inside supernovas, and to determine mass numbers corresponding to the "peaks" of abundance of elements in Galaxy, numerically computed in [24]. In addition, it turned out to be possible to get estimates of time formation and typical sizes of neutron stars at supernova outbursts. In the field of mesoscopics this approach is valid and reasonably effective for materials with profound strong interatomic bonds: carbon nanostructures with covalent bonds as well as for nanostructured particles of light actinides with strong approach of atoms [15]. The expediency of pursuing development of asymptotic models [15] in the field of mesoscopics is connected with both the creation of ultra-hard carbon materials and the problem of phase stability and plutonium polymorphic transformations.

Below are arguments for substantiating and more detailed presenting of formalism of a general kinetic approach to description of formation and growth of objects. Also, it is reasonable to present the expanded fields of application for the proposed asymptotic models.

\section{General Kinetic Approach}

Let us consider formation and growth of compact clusters in conservative stochastic systems defined as assemblies of total-mass-limited quantum objects interacting with each other randomly. We will describe the process of the irreversible aggregation of objects using the concept of distribution density wave $\varphi(x, y)$ in the space of cluster sizes $a$. The wave propagates with the time $t$ toward an increase in the cluster size. Such one-dimensional approach allows one not to take into account deviation of a geometric shape of the object from the ideal one. On the assumption of the above the universal relation for a wave packet half-width and a spectral line half-width for coherent processes one can write down the following uncertainty relation for a coordinate and a momentum in the space of cluster sizes [25]:

$$
\Delta a \cdot \Delta p \cong \frac{\hbar}{2} .
$$

Here, $\Delta p \sim p=m \Delta a / \Delta t$ is momentum uncertainty, $m$ is cluster mass, $\hbar$ is reduced Planck constant. Momentum uncertainty is equal to the momentum itself in the order of magnitude, i.e., interaction of objects either occurs or does not occur. Physical meaning of relation (1) is in the fact that during the time interval $\Delta t$ of elementary (single) act of interaction between objects the exact cluster size cannot be determined until this interaction is finished either through capture of one object by another or their partial or complete disruption, or elastic scattering. It is connected with the fact that unless the elementary act is finished, it is impossible to determine to which object each of the interacting surface elements (nucleons in case of nuclear matter and atoms in case of crystal mesostructures) is assigned. The sign of approximate equality reflects quasi-coherent behavior of considered systems specified by possible fluctuations connected with the specific character of high intensive processes (high-velocity collisions breaking the initial structure of objects; inharmoniousness of atomic oscillations at environment high temperature).

Formally, it follows from the relation (1) that $\Delta a \propto \sqrt{\Delta t}$, i.e., an instantaneous velocity of growth of object size is infinitely large:

$$
\mathrm{d} a / \mathrm{d} t=\lim _{\Delta t \rightarrow 0} \Delta a / \Delta t \propto \lim _{\Delta t \rightarrow 0} 1 / \sqrt{\Delta t}=\infty
$$

Because of random character of aggregation process of investigated objects the analytical derivative $\mathrm{d} a / \mathrm{d} t$ cannot be determined, and the index of the fractional derivative is equal to $1 / 2$. To describe such a stochastic process using any analytical function $F(a)$ one should use a differential of the type $\mathrm{d} F=F^{\prime} \mathrm{d} a+(1 / 2) F^{\prime \prime}(\mathrm{d} a)^{2}$ [26].

In the closed system of clusters the distribution density function $\varphi(a, t)$ satisfies the condition of total mass conservation:

$$
M_{\text {nucl }}(t)+M_{c l}(t)=M_{\text {nucl }}(t)+\int_{0}^{a_{\max }} \varphi(a, t) m(a, t) \mathrm{d} a=\text { const },
$$

where $M_{\text {nucl }}(t), M_{c l}(t)$ are current total masses of seeds and clusters, respectively, $a_{\max }$ is the maximum cluster size in the system; and the mass of volumetric close-packed cluster can be defined either as $m=m_{0}\left(a / a_{0}\right)^{3}$, 
where $a_{0}, m_{0}$ are size and mass of a cubic shape seed, or as $m=\alpha \rho a^{3}$, where $\rho$ is cluster material density, $\alpha$ is geometric factor (for a cube $\alpha=1$, for a sphere $\alpha=\pi / 6$ ). At the end of aggregation process the total mass of seeds is infinitely small, and hence, the first term in conservation Equation (2) can be neglected. Total cluster mass can be considered as integral of motion, i.e., one can consider that the mass increment $\Delta M_{c l}(t)$ at $t \rightarrow t_{f}$ tends to zero ( $t_{f}$ is the time instant of aggregation completion). Revealing increment of a certain integral in expression (2), we obtain

$$
\lim _{t \rightarrow t_{f}} \Delta M_{c l}=\lim _{t \rightarrow t_{f}} \Delta \int_{0}^{a_{\max }} \varphi(a, t) m(a, t) \mathrm{d} a=\alpha \rho \int_{0}^{a_{\max }}\left\{\lim _{t \rightarrow t_{f}} \Delta\left[a^{3} \varphi(a, t)\right]\right\} \mathrm{d} a=0 .
$$

Since at the end of the process cluster sizes and quantities are large comparing to those of seeds, the "simultaneous" failure of a significant number of clusters at their interaction with seeds is highly unlikely. Frozen distribution of clusters in sizes is established in the system, and an integrand in expression (3) does not change its sign, at least, within the time interval $\left(t_{f}-\Delta t, t_{f}\right)$. Hence from (3) it follows that

$$
\lim _{t \rightarrow t_{f}} \Delta\left[a^{3} \varphi(a, t)\right]=0
$$

Introducing the dimensionless variable $a / a_{0}$ and revealing the last expression according to rules [26], for the above differential $\mathrm{d} F$ we obtain:

$$
\mathrm{d} \ln \varphi=-3\left\{\mathrm{~d} \ln \left(a / a_{0}\right)+\frac{3}{2}\left[\mathrm{~d}\left(a / a_{0}\right) /\left(a / a_{0}\right)\right]^{2}\right\}, t \rightarrow t_{f} .
$$

With high $a \gg a_{0}$ the second term in braces can be neglected. It is finally found that the density function of size distribution of large clusters at the end of the aggregation process $\varphi_{\text {fin }}$ can be approximated by the following relationship:

$$
\varphi_{\text {fin }} \propto a^{-3} \propto m^{-1}, a \gg a_{0}, t \rightarrow t_{f} .
$$

This asymptotic kind of distribution obtained using uncertainty relation (1) is not connected with the initial distribution $\varphi_{0}(a)$, and does not depend on the type of a kinetic equation for $\varphi$. Distribution (4) can be regarded as "invariant" of any kinetic equation describing behavior of clusters with quantum properties.

In accordance with the above kind of analytic function differential, evolution of distribution density function $\varphi(a, t)$ during a stochastic process of aggregation can be described in diffusion approximation using FokkerPlanck equation written for the space of cluster sizes $a$ :

$$
\frac{\partial \varphi(a, t)}{\partial t}+\frac{\partial}{\partial a}[v \varphi(a, t)]-\frac{1}{2} \frac{\partial^{2}}{\partial a^{2}}[\eta \varphi(a, t)]=0
$$

Here, $v=\langle\mathrm{d} a\rangle / \mathrm{d} t, \eta=\left\langle(\mathrm{d} a)^{2}\right\rangle / \mathrm{d} t$ is the average rate of kinematic transport of $\varphi$ and diffusion coefficient in the space $a$, respectively. To linearize this equation and obtain approximate solutions we will accept that the average rate of kinematic transfer of $\varphi$ matches in order of magnitude the growth rate of an average cluster size:

$$
v=\langle\mathrm{d} a\rangle / \mathrm{d} t \cong \mathrm{d}\langle a\rangle / \mathrm{d} t
$$

In accordance with the ratio (1) the diffusion coefficient can be written as:

$$
\eta \cong \hbar / 2 m=\hbar / 2 m_{0}\left(a / a_{0}\right)^{3} \text {. }
$$

We introduce dimensionless variables $\xi=a / a_{0}, \tau=t / t_{i}$, where $t_{i}$ is a typical time scale of object interactions determined by their nature and aggregation mechanism. Then, taking into account the accepted assumptions, Equation (5) takes the following form:

$$
\frac{\partial \varphi(\xi, \tau)}{\partial \tau}+\Psi(\tau) \frac{\partial \varphi(\xi, \tau)}{\partial \xi}-\frac{1}{2} \beta \frac{\partial^{2}}{\partial \xi^{2}}\left[\varphi(\xi, \tau) \cdot \xi^{-3}\right]=0 .
$$

Here, $\Psi(\tau)=\mathrm{d}\langle\xi\rangle / \mathrm{d} \tau$ is dimensionless growth rate of average size of clusters, and $\beta \cong \hbar t_{i} / 2 m_{0} a_{0}^{2}$. To estimate the value $\beta$ we will draw on ratio (1) and the mass conservation law in an elementary process of inte- 
raction between a cluster and a seed in the assumption that seed mass gets equally "smeared" on a cluster surface:

$$
\rho a^{3}+\rho a_{0}^{3}=\rho(a+\Delta a)^{3} .
$$

Here, $\rho$ is the density of cluster matter. From the conditions $a_{0}<a, \Delta a \ll a$ we will find that $\Delta a \cong a_{0}^{3} / 3 a^{2}$. Then, from ratio (1) we obtain that $\beta \cong(1 / 81)\left(a_{0} / a\right) \ll 1$. Hence $\beta$ is a small parameter.

Revealing the derivative in the third term of Equation (6), we obtain the following equation:

$$
\frac{\partial \varphi}{\partial \tau}+\Psi \frac{\partial \varphi}{\partial \xi}-\frac{\beta}{2 \xi^{3}} \frac{\partial^{2} \varphi}{\partial \xi^{2}}+\frac{3 \beta}{\xi^{4}} \frac{\partial \varphi}{\partial \xi}-\frac{6 \beta}{\xi^{5}} \varphi=0 .
$$

The study into asymptotic properties of Equation (7) performed in [25] has shown the following. With high $\xi \gg 1$, the law of average size growth becomes:

$$
\langle a\rangle \cong a_{0} \mathrm{~K}\left(\frac{t}{t_{i}}\right)^{\mathrm{Z}} .
$$

The constants K, Z are determined by an expression coming from uncertainty relation (1) transcribed for an average size of clusters:

$$
\langle m\rangle \frac{(\Delta\langle a\rangle)^{2}}{\Delta t} \cong \frac{\hbar}{2} .
$$

At $\Delta t=t_{i}$, we obtain the following expression:

$$
\mathrm{Z}^{2} \cdot \mathrm{K}^{2 / \mathrm{Z}} \cong \frac{\hbar t_{i}}{2 m_{0} a_{0}^{2}}\left(\frac{\langle a\rangle}{a_{0}}\right)^{\frac{2}{\mathrm{Z}}-5}
$$

Value $\mathrm{Z}$ is given from the condition $\mathrm{K} \equiv$ const, i.e., a right-hand portion of expression (9) should not depend on $\langle a\rangle$. Specific values of constants in law (8) are related with the value $t_{i}$, i.e., they are determined by a mode and mechanism of object interactions in a closed system.

Partial solution of Equation (7) at $\xi \gg 1$ looks like:

$$
\varphi_{\lambda}=C(\lambda) \exp \left[-\lambda^{2}(\xi-\langle\xi\rangle)\right],\langle\xi\rangle \cong \mathrm{K} \tau^{\mathrm{Z}}
$$

Here, $C(\lambda)$ is a function of the arbitrary real number $\lambda$. General solution fitting the law of mass conservation and asymptotic distribution (4) (with $C(\lambda)=\mathrm{B} \lambda^{5}, \mathrm{~B} \equiv$ const), can be written as:

$$
\varphi(\xi, \tau)=\int_{-\infty}^{\infty} C(\lambda) \exp \left[-\lambda^{2}(\xi-\langle\xi\rangle)\right] \mathrm{d} \lambda=\mathrm{B} 2 ! /(\xi-\langle\xi\rangle)^{3}, \quad \xi \gg 1 .
$$

Comparing this expression with distribution (4) one can conclude that at $t \rightarrow t_{f}$ the condition $\langle a\rangle_{f i n} \ll a_{\max }$ is to be satisfied. At the end of the process of object irreversible aggregation as a result of interaction between large clusters and small seeds in a stochastic conservative system the distribution density of large clusters is in inverse proportion to their masses, and average size of clusters is significantly less than the final maximum size.

Approximate view of $\varphi$-function close to maximum of distribution density in size curve can be determined by omitting in Equation (7) terms with the first variable derivative $\xi$, since in small neighborhood of local maximum the analytical function changes poorly: $\partial \varphi / \partial \xi \cong 0$. Having written the function $\varphi$ as a product of space and time factors and having solved the equation for the spatial factor P by Ventzel-Kramers-Brillouin method [16], we obtain the following expression:

$$
\mathrm{P}=\frac{(\beta / 2)^{1 / 4}(\xi / \lambda)^{1 / 2}}{\left(c^{2}+\xi^{5}\right)^{1 / 4}} \sin \left\{\frac{2 \lambda}{5}\left(\frac{2}{\beta}\right)^{1 / 2}\left[\sqrt{c^{2}+\xi_{n}^{5}}-\frac{c}{2} \ln \frac{\sqrt{c^{2}+\xi_{n}^{5}}+c}{\sqrt{c^{2}+\xi_{n}^{5}}-c}\right]+\text { const }\right\},
$$

where, $c^{2} \equiv 6 \beta / \lambda^{2}$. The presence of the oscillating factors in $\{\ldots\}$ in the expression (12) means that function $\mathrm{P}$, in principle, can have variety of local maximums with $\xi_{n}$ coordinates. What this means is existence of variety of the most probable (stable) sizes of clusters. The constant in the expression (12) is found from the condition 
that the first maximum $(n=0)$ corresponds to seeds $\left(\xi_{0}=1\right)$. Since $\beta \ll 1, \lambda>1$, the parameter $c^{2} \ll 1$. Then the condition of maximums looks like:

$$
\frac{2 \sqrt{2}}{5} \frac{\lambda}{\sqrt{\beta}}\left(\xi_{n}^{5 / 2}-1\right) \cong n \pi, n=1,2,3, \cdots
$$

The condition of $\mathrm{P}$-function "zeros" with consideration for small $c^{2}$ and $c$ values looks as:

$$
\frac{2 \sqrt{2}}{5} \frac{\lambda}{\sqrt{\beta}} \xi_{k}^{5 / 2}+\text { const' }^{\prime} \cong \pi k, k=1,2,3, \cdots
$$

The presence of many "zeros" in P-function means the existence of unstable sizes of clusters.

We consider size distribution of "small" clusters for which kinematic transport of distribution density is expressed weaker than diffusion. Having omitted in Equation (7) the second term responsible for transport and having solved the obtained equation, we will get the following expression for a space factor:

$$
\mathrm{P}=\xi^{3}-\frac{\lambda^{2}}{20} \xi^{8}
$$

Function maximum condition (15) takes the form:

$$
\mathrm{P}^{\prime}=3 \xi^{2}-\frac{8 \lambda^{2}}{20} \xi^{7}=0, \mathrm{P}^{\prime \prime}=6 \xi-\frac{56 \lambda^{2}}{20} \xi^{6}<0 .
$$

"Joining" of maximum condition (16) with the first maximum condition in expression (12) $n=0, \xi_{0}=1$ allows one to determine the arbitrary constant $\lambda$ :

$$
3-\frac{8}{20} \lambda^{2}=0 \Rightarrow \lambda^{2}=\frac{15}{2}, \mathrm{P}^{\prime \prime}=-21<0 .
$$

With the knowledge of $\lambda$, it is possible to determine the coordinate of $\mathrm{P}$-function first "zero":

$$
\mathrm{P}=0 \Rightarrow \xi=\left(\frac{20}{\lambda^{2}}\right)^{1 / 5}=1.216728684 .
$$

"Joining” of expressions (14) and (18) with $k=0$ makes it possible to find a constant value in expression (14) and write the following condition for coordinates of function P "zeros":

$$
\frac{2 \sqrt{2}}{5} \frac{\lambda}{\sqrt{\beta}}\left(\xi_{k}^{5 / 2}-\frac{\sqrt{20}}{\lambda}\right) \cong \pi k, k=1,3,5, \cdots
$$

What this means is existence of unstable clusters with corresponding to expression (19) sizes, the first "unstable" size in this case is equal to $\xi=1.216728684$.

Expression (15) shows that with $\xi<1$ the distribution density of seed failure products is approximately proportional to their masses. This is incompliance with the concept that disintegration of seeds into reasonably large fragments is more likely (advantageous in energy), than complete failure into individual components.

The linearization of Fokker-Planck kinetic Equation (5) allows one to establish basic properties of cluster size distribution density function. Expressions (8), (9) can be used for finding laws of time-dependent average size growth of large clusters $\left(\langle a\rangle \gg a_{0}\right)$. We consider two limiting growth modes: 1) seed small flux, when each of the seeds has time to occupy its energy advantageous place on cluster surface before interaction with the following seed begins; 2 ) seed large flux, when seeds affect the cluster almost simultaneously which is equivalent to cluster collision. In the first case $t_{i}$ parameter does not depend on a cluster size and the law of growth can be written as:

$$
\langle a\rangle \cong(5 / 2 \sqrt{2})^{2 / 5} a_{0}\left(t / t_{1}\right)^{2 / 5}, t_{1}=(2 \sqrt{2} / 5) a_{0} \sqrt{m_{0} t_{i} / \hbar} .
$$

Here $t_{1}$ is the time unit in the mode of seed small flux. In case of seed large flux we accept the $t_{i}$ parameter equal to the perturbation back-and-forth travel time in a cluster: $t_{i}=2\langle a\rangle / c_{0}$, where $c_{0}$ is velocity of perturbation spread in cluster substance (sonic velocity). Then the law of growth can be written as:

$$
\langle a\rangle \cong(3 / 2)^{1 / 3} a_{0}\left(t / t_{2}\right)^{1 / 3}, t_{2}=a_{0} \sqrt{a_{0} m_{0} / \hbar c_{0}} .
$$


Here $t_{2}$ is the time unit in the seed large flux mode (cluster collision).

It should be noted that laws of growth for average size objects can be deduced directly from uncertainty relation (1'), if to substitute $\Delta t=t_{i}$ in it, determine $\Delta\langle a\rangle$, and then to solve the differential equation $\mathrm{d}\langle a\rangle / \mathrm{d} t \approx \Delta\langle a\rangle / \Delta t=\Delta\langle a\rangle / t_{i}$ with zero initial condition. With the seed small flux we obtain the following chain:

$$
\Delta\langle a\rangle \cong \sqrt{\frac{\hbar t_{i}}{2 m_{0}}} \frac{a_{0}^{3 / 2}}{\langle a\rangle^{3 / 2}} \rightarrow \frac{\Delta\langle a\rangle}{\Delta t} \cong \sqrt{\frac{\hbar}{2 m_{0} t_{i}}} \frac{a_{0}^{3 / 2}}{\langle a\rangle^{3 / 2}} \cong \frac{\mathrm{d}\langle a\rangle}{\mathrm{d} t} \rightarrow\langle a\rangle^{3 / 2} \mathrm{~d}\langle a\rangle \cong\left(\frac{\hbar a_{0}^{3 / 2}}{2 m_{0} t_{i}}\right)^{1 / 2} \mathrm{~d} t .
$$

Solving the last differential relation in quadratures, we will obtain the following expression:

$$
\langle a\rangle \cong\left(\frac{25 \hbar a_{0}^{3}}{8 m_{0} t_{i}}\right)^{1 / 5} t^{2 / 5}
$$

This exactly agrees with law (20), if to reveal the $t_{1}$ parameter there. Similarly, with $\Delta t=2\langle a\rangle / c_{0}$ we obtain:

$$
\langle a\rangle \cong\left(\frac{3}{2}\right)^{1 / 3}\left(\frac{\hbar c_{0} a_{0}^{3}}{m_{0}}\right)^{1 / 6} t^{1 / 3} .
$$

This agrees with law (21). In the first approximation one can assume that time-dependent laws of growth (22), (23) for average size of objects are invariants of any kinetic equation describing aggregation of clusters with quantum properties.

In number of problems connected with investigations of high-intensive processes with high energy (for example, at high temperatures of crystal objects) it is reasonable to determine the value of $\Delta t$ time interval of elementary interaction act based on the "energy-time" uncertainty relation:

$$
\Delta t \cong \hbar / \Delta E \text {. }
$$

Here, $\Delta E$ is energy level width of an isolated excited state of a quantum mechanical system. Substituting expression (24) in relation (1'), one can write the following chain of differential relations:

$$
\frac{\Delta\langle a\rangle}{\Delta t} \cong\left(\frac{a_{0}^{3} \Delta E}{2 m_{0}}\right)^{1 / 2} \frac{1}{\langle a\rangle^{3 / 2}} \cong \frac{\mathrm{d}\langle a\rangle}{\mathrm{d} t} \rightarrow\langle a\rangle^{3 / 2} \mathrm{~d}\langle a\rangle \cong\left(\frac{a_{0}^{3} \Delta E}{2 m_{0}}\right)^{1 / 2} \mathrm{~d} t .
$$

The $\Delta E$ value is determined by nature of objects and a process mode. For example, in case of phonon excitation of mesoscopic crystals at high temperatures $k_{B} T \gg \hbar \omega_{\text {char }}$ ( $k_{B}$ is Boltzmann constant, $\omega_{\text {char }}$ is characteristic frequency), the $\Delta E$ value is directly proportional to $\mathrm{N}$ number of atoms, covered by phonon excitations: $\Delta E \cong 3 N k_{B} T$ [27]. The value $\mathrm{N}$ can depend on the average size $\langle a\rangle$ of an excitation region. Having solved differential relation (25) in quadratures one can find approximate laws of average size $\langle a\rangle$ growth with time in high-intensive processes of object aggregation followed by multiple production and elimination of phonons at high temperatures.

We consider an issue of upper limits of object sizes. From uncertainty relation (1) and mass conservation condition in elementary interaction between a large cluster and a seed, when $\Delta a \cong a_{0}^{3} / 3 a^{2}$, we obtain the following expression for the maximum object size

$$
a_{\max } \cong \frac{2}{9} \alpha \frac{\rho}{\hbar} \frac{a_{0}^{6}}{\Delta t_{\min }} \cong \frac{2}{9} \frac{m_{0}}{\hbar} \frac{a_{0}^{3}}{\Delta t_{\min }}
$$

Here, $\Delta t_{\min }$ is a minimum time interval of an elementary act of object interaction determined by physical nature of the process.

\section{Subatomic and Astrophysical Objects}

In this section various nuclear phenomena are viewed from unified point of view as processes of formation and growth of compact clusters with pronounced collective quantum properties (strong interaction) in a closed sto- 
chastic nucleon system in an excited state. As the excited closed system one can consider a mother nucleus, or two contacting nuclei after their collision (double system). At the initial time such system consists of nuclear matter seeds: alpha-particles and (or) their fragments-tritons and deuterons. As a result of random interaction of these seeds their coalescence takes place. Compact accumulations of nuclear continuum (clusters) are formed. Nucleon oscillations on the surface of adjoining "nuclear" clusters having temporarily (virtually) broken external bonds can result in mutual compensation of these unused bonds. As a result the adjoining clusters consolidate, and larger nuclear objects with various masses (mass numbers $A$ ) are formed. Due to Coulomb repulsion these objects can escape from the region of interaction. The proposed model can be applied to the following problems: 1) calculations of the most probable mass numbers $A$ of clusters within the entire range of the existing nuclide systematization [28] [29]; 2) computed estimation of a discrete spectrum of the most probable numbers $A$, typical for cluster radioactivity investigated in [30] [31]; 3) discovering the most probable average mass numbers $\langle A\rangle$ of clusters formed in processes of spontaneous nuclear fission [32] [33]; 4) determination of the most probable values $A$ and evaluation of the average value $\langle A\rangle$ of superheavy clusters formed in processes of nucleosynthesis in stars [24] [34]. Only fast intranuclear processes ( $r$-processes) that go during lifetime of excited states are considered. These states are given by the energy of oscillatory and rotational levels determined in shell model of nucleus. The time of processes of approaching equilibrium is within the interval between the time of direct nuclear reactions (with typical nuclear time of $\left.\sim 10^{-22} \mathrm{~s}\right)$ and the lifetime of a compound nucleus $\left(10^{-16}-10^{-15} \mathrm{~s}\right.$ [29] [35]-[39]). In addition, there are some attempts to determine separately typical formation times and nuclear matter cluster sizes in astrophysical scales, in particular, for neutron stars.

The basis for analytical description of the processes under consideration is a phenomenological concept (given in Section 2) of the object distribution density wave $\varphi(a, t)$ in space of their sizes $a$. Such one-dimensional approach allows one not to take into account deviation of a real geometric shape of nucleus from spherical one. Evolution of function $\varphi(a, t)$ during a stochastic process of object aggregation can be described in diffusion approximation using Fokker-Planck Equation (5) written for the space of cluster sizes. To determine the most probable mass numbers we will use expression (12) with an arbitrary real number $\lambda=\sqrt{15 / 2}$ found out in Section 2 and known expressions [29] [39] connecting the nucleus radius $R=a / 2$ and the mass number:

$$
R \cong r_{0} A^{1 / 3}
$$

where $r_{0}=1.3 \mathrm{fm}$ is a typical parameter of strong interaction. If one accepts that this expression is approximately fulfilled for seeds as well, then from Equation (12) it is possible to obtain the following expression for the most probable mass numbers $A_{n:}$

$$
A_{n} \cong A_{0}\left(\frac{5}{2 \sqrt{2}} \frac{\sqrt{\beta}}{\lambda} \pi n+1\right)^{6 / 5}, \quad n=1,2,3, \cdots
$$

Here, $A_{0}$ is seed mass number, and the parameter $\beta \cong \hbar t_{i} / 2 m_{0} a_{0}^{2}$ is determined by a seed mass and size and the typical time scale $t_{i}$ of interaction between objects. It is reasonable to accept as such scale a period of high-frequency nucleon oscillations in a nucleus. This parameter can be determined as $t_{i}=T_{h f}=2 r_{0} / c_{0}=5 \times 10^{-23} \mathrm{~s}$, where $c_{0}=5 \times 10^{7} \mathrm{~m} \cdot \mathrm{s}^{-1}$ is an average thermal velocity of nucleons in degenerate Fermi gas determined by the known value of average kinetic energy of ideal Fermi gas per a nucleon, equal to $E_{F}=22 \mathrm{MeV}$ [39].

To find typical average sizes of cluster nuclides we will use expressions (22) and (23) obtained in Section 2 and transcribe them as follows:

$$
\begin{gathered}
\langle a\rangle_{1} \cong(5 / 2 \sqrt{2})^{2 / 5}\left(6 \hbar / \pi \rho t_{i}\right)^{1 / 5} t^{2 / 5}, \\
\langle a\rangle_{2} \cong(3 / 2)^{1 / 3}\left(6 \hbar c_{0} / \pi \rho\right)^{1 / 6} t^{1 / 3}
\end{gathered}
$$

Here, $\rho$ is density of nuclear matter. In order to eliminate the parameter $a_{0}$ from equations, it was accepted that $m_{0} \cong(\pi / 6) \rho a_{0}^{3}$. The absence of a seed size in (29) and (30) reflects an asymptotic stage of the process, when the system "forgets" about initial conditions and its evolution goes on according to internal dynamics of nucleus. This dynamics is connected with excitation of oscillatory and rotational energy levels.

General time of the process is determined by shell energy level $\Gamma$ using Heisenberg rule [35] [36]:

$$
\tau=\hbar / \Gamma \text {. }
$$


It is correct for isolated excitation of level in quantum system.

Verification of the proposed formalism can be implemented by the example of "rigid" (deep inelastic) processes involving hadrons described in [40] [41]. We consider these processes as the following chain of events: head-on collision of "nucleon-nucleon" or "lepton-nucleon" $\rightarrow$ quark knocking-out from gluon cloud $\rightarrow$ random interactions in intra-hadron medium $\rightarrow$ new particle formation (hadron jets). In [23] [25] the new particles are considered as clusters consisting of intra-hadron medium components (partons): quarks, gluons, quark-antiquark couples. A condition of generation of new particles can be written as:

$$
\left(M_{\text {elem }}+M_{c l}\right) c^{2}=M_{0} c^{2} .
$$

Here, $M_{\text {elem }} c^{2}$ is total energy of partons, $M_{c l} c^{2}$ is total energy of new particles, $M_{0} c^{2}$ is total energy of two original colliding particles. At the end of this process all the partons transformed into bound states, $M_{\text {elem }}=0$, therefore relation (3) is fulfilled, and the proposed in Section 2 stochastic approach is applicable. Then law (21) for describing the evolution of cluster average size provides the following relation between length $a_{\text {unit }}$, time $t_{\text {unit }}$ and mass $m_{\text {unit }}$ units under deep inelastic interaction of fundamental particles:

$$
m_{\text {unit }} a_{\text {unit }}^{3} / t_{\text {unit }}^{2}=\hbar c \text {. }
$$

Here $c$ is the maximum velocity of interaction propagation in hadron medium: the speed of light in vacuum. Based on relation (32) and the data from [19] for "conventional" quark masses ( $u$ - and $d$ - quarks) deep inside hadrons and for typical distances, one can obtain the following estimation of the time unit $t_{u n i t}^{a f}$ for current quarks $(c q)$ in the state of asymptotic freedom $(a f)$ :

$$
a_{\text {unit }}^{a f} \sim 10^{-16} \mathrm{~m}, m_{\text {unit }} \sim m_{u}^{c q} \approx 5 \mathrm{MeV}, m_{\text {unit }} \sim m_{d}^{c q} \approx 7 \mathrm{MeV} ; \Rightarrow t_{\text {unit }}^{a f} \sim 10^{-26} \mathrm{s.}
$$

The quark transition time $t_{\text {trans }}$ from asymptotic freedom to a bound state (confinement) inside a hadron is evaluated according to Formula (21) as $t_{\text {trans }} \sim t_{\text {unit }}^{a f}\left(a_{\text {unit }}^{\text {conf }} / a_{\text {unit }}^{a f}\right)^{3} \sim 10^{-23} \mathrm{~s} \quad\left(a_{\text {unit }}^{\text {conf }} \sim 10^{-15} \mathrm{~m}\right)$. The obtained value corresponds to the time scale of strong interaction.

In processes considered in [40] the minimum value of length unit is $a_{u n i t}^{\min } \sim 10^{-18} \mathrm{~m}$. It can be considered as the top estimation of current quark size. Then it goes from relation (32) that $t_{\text {unit }}^{\min } \sim 10^{-29} \mathrm{~s}$, and the time $t_{\text {hadr }}^{\text {form }}$ for formation of hadrons having an average size of $\langle a\rangle \sim 10^{-15} \mathrm{~m}$, in accordance with Formula (21), is $t_{\text {hadr }}^{\text {form }} \approx t_{\text {unit }}^{\min }\left(\langle a\rangle / a_{\text {unit }}^{\min }\right)^{3} \sim 10^{-20} \mathrm{~s}$. This value is significantly higher than the life time of unstable hadrons ("resonances") $t_{\text {life }}^{\text {res }} \leq 10^{-21} \mathrm{~s}: t_{\text {hadr }}^{\text {form }} \gg t_{\text {life }}^{\text {res }}$. This reflects formation of stable hadron jets in described processes of catastrophic collisions.

If in accordance with the results of [42], we accept that perturbation propagation rate in quark-gluon medium is equal to sonic speeds $c_{\mathrm{s}}=0.3 \mathrm{c}$ and $0.57 \mathrm{c}$, then in the above estimates of the time units (the time of quark transformation into a bound state and the time of hadron formation) factors 1.8 and 1.3 will appear. These corrections do not change the estimate of strong interaction time scale and increase the inequality $t_{\text {hadr }}^{\text {form }} \gg t_{\text {life }}^{\text {res }}$ reflecting the formation of stable hadron jets.

The asymptotic distribution $\varphi \propto \mathrm{m}^{-1}$ means that: 1) probability of nucleon fragmentation in deep inelastic scattering is higher than probability of their conservation; 2) the number of pions in hadron jet is 1.5 times higher that the number of nucleons. These conclusions are in compliance with concepts of [40] about nature of rigid processes. In particular, at proton frontal collision the following reaction takes place: $p p \rightarrow \pi^{0} \pi^{+} \pi^{+} n n$. One can see that there are 3 pions and 2 neutrons in the formed hadron jet.

Consequently, the proposed formalism allows adequate description of an asymptotic form of well-known accidents in the world of elementary particles. This gives grounds to try to apply it in solving the above described problems of intranuclear processes description.

Values $A_{n}$ calculated in [14] [25] with the help of Formula (28) with three types of seeds correspond to the mass numbers of the nuclides and their isotopes over the currently known range [28] [29], including the transfermium elements. In addition, calculated mass numbers of cluster nuclides are also in compliance with standard concepts [29] [43] on isotope "stability valley". It should be kept in mind that the calculated clusters are formed deep in the sea of nuclear matter and not in particular reactions. In escaping from either the parent nucleus or the double system clusters can emit or capture individual nucleons and seeds of nuclear matter. 
The established correspondence allows the characteristic values for various processes of approaching equilibrium (cluster radioactivity, spontaneous fission, synthesis of heavy and superheavy elements) to be extracted from the above values.

Calculated mass numbers of clusters formed inside heavy nuclei in processes of cluster radioactivity make up the following discrete spectrum: $A=6,7,9,11-16,18,20,24,26,28,30,32,34,37-40$. In experiments on cluster radioactivity the values $A=14,24,28,32$ were recorded. Under discussion is the possibility of recording the outgoing clusters with mass numbers close to stated predicted spectrum (e.g., see [22] [30] [31]).

In the field of spontaneous fission it is possible to single out two sets of the most probable cluster mass numbers, each being repeated for two or three seed types: $A_{1}=72,79,84,88,103,108$ и $A_{2}=126,134,139,142$, $147,155,158$. The arithmetic means for the two sets of mass numbers are equal to $\bar{A}_{1} \cong 90$ и $\bar{A}_{2} \cong 140$. The obtained values correspond to the coordinates of the maxima of the total nuclear fission yields [32] [33]. It is possible to describe spontaneous nuclear fission as a result of excitation of the first rotational level with the energy $\Gamma_{\text {rot }}=100 \mathrm{keV}$ using the Formulae (27) and (30). The total time of an intranuclear process corresponding to the given value $\Gamma_{\text {rot }}$, is equal to $\tau_{\text {rot }}=6.287 \times 10^{-21} \mathrm{~s}$, and the Formulae (27) and (30) determine an average mass number of light fragments as $\langle A\rangle_{\text {light }}=100$. An average mass number of heavy fragments $\langle A\rangle_{\text {heavy }}$ is determined from the condition of mass conservation $\langle A\rangle_{\text {mat }}=\langle A\rangle_{\text {light }}+\langle A\rangle_{\text {heavy }} ; A_{\text {mat }}$ is mass number of parent nucleus. In case of light actinides (Th, $\mathrm{U}$ ) we get $\langle A\rangle_{\text {heavy }}=135-138$. In diagrams [32] [33] of fragment mass distribution in spontaneous heavy nuclear fission $(A=235,238)$ an average mass number of lighter fragments is 90-100, an average mass number of heavier fragments is about 140.

If one assumes that fast nucleosynthesis in stars occurs as a result of transition of nucleon system from high-frequency vibration level with the period $t_{i}=5 \times 10^{-23} \mathrm{~s}$ to the first rotational level with the typical energy $100 \mathrm{keV}$ during the lifetime of this level $\tau_{\text {rot }}=6.287 \times 10^{-21} \mathrm{~s}$, then Formulae (27) and (29) provide the following estimate of an average mass number of superheavy elements: $\langle A\rangle_{\text {superheavy }}=330$. The obtained value agrees with the prediction from [24] about possibility of nucleus formations with $A>300$ in neutron stars. As for the induced fission as the process competing with nucleosynthesis, in this case one can accept as a typical scale $t_{i}$ the sonic wave travel time $t_{0}$ in a nucleus formed as a result of nucleosynthesis: $t_{0}=a / c_{0}$. With $A=330, c_{0}=5$ $\times 10^{7} \mathrm{~m} \cdot \mathrm{s}^{-1}$, we obtain $t_{i}=3.6 \times 10^{-22} \mathrm{~s}$. Then from Formulae (27) and (29) we obtain that with the above lifetime of the first rotational level $6.287 \times 10^{-21} \mathrm{~s}$ the average mass number of light fragments of superheavy elements is $\langle A\rangle_{\text {light }}=100$. In compliance with the mass conservation law the average mass number of heavy fragments is equal to $\langle A\rangle_{\text {light }}=230$. The obtained values correspond approximately to the first and the last peaks of final abundance of elements in Galaxy [34]. This compliance gives grounds to expect that the above estimate of the average mass number of superheavy elements that can be formed as a result of nucleosynthesis in supernovas and neutron stars is reliable.

One can try to estimate a mass number of the final nuclide $A_{\text {end }}$ formed in $r$-processes of nucleosynthesis in stars, if he uses expression (26) for the maximum cluster size in a closed system and the known expression (27) for the mass-number-dependence of nucleus radius. If as $\Delta t_{\min }$ in (26) we assume the travel time of an electromagnetic wave over a nucleus equal to $a / c$ ( $c$ is the velocity of light in vacuum), then from espressions (26) and (27) we will obtain the following equation for a mass number of the final nuclide:

$$
A_{\text {end }} \cong\left(\frac{\pi}{27}\right)^{3 / 2}\left(\frac{\rho c}{\hbar}\right)^{3 / 2}\left(4 r_{0}^{2}\right)^{3} A_{0}^{3}
$$

This, with alpha particle $\left(A_{0}=4\right)$ taken as a seed, yields to $A_{\text {end }}=470$. According to the expression (28) mass number 470 corresponds to triton as the seed. This value is by approximately $15 \%$ higher than value 408 responsible for hypothetical continuation of $2 \beta$-stability line [43]. Hence the obtained estimate is in compliance with the known systematization of nuclei.

The above time $\tau_{\text {rot }} \sim 10^{-20} \mathrm{~s}$ of intranuclear processes of nucleosynthesis and fission is negligible as compared to the given in [24] [34] times $\tau_{\text {yield }}=0.3-2 \mathrm{~s}$, when performing numerical simulation of nucleosynthesis and fission yield in astrophysical phenomena. In accordance with concepts about supernovas and neutron stars published in encyclopedia [44] [45], the times $\tau_{\text {yield }}$ are connected with hydrodynamic processes of neutron star formations in supernova outbursts as a result of gravitational compression of the star central region.

It is possible to determine times of neutron star formations by an analytical way. For this purpose we will use the analogy with the concept [19] on introduction of phenomenological constants. From considerations of dimensionality and by analogy with the notion of Planck mass $m_{p}=\left(\hbar c / G_{N}\right)^{1 / 2}$, determined through the action 
constant $\hbar$ in microcosm, the gravitation constant $G_{N}$ and the light speed $c$, we will write the following expression for the action constant $K_{G}$ in the "world" of collapsing stars:

$$
K_{G}=\frac{\mathrm{M}_{C h}^{2} G_{N}}{C}=1.72 \times 10^{42} \mathrm{~J} \cdot \mathrm{s}
$$

Here $\mathrm{M}_{C h}=1.4 \mathrm{M}_{S}$ is the Chandrasekhar critical mass [45] [46] above which the star gravitational compression begins, $M_{S}$ is the mass of the Sun. When postulating the uncertainty principle in gravitational processes by analogy with Heisenberg "energy-time" uncertainty relation for microcosm [46], one can write the following expression for the lifetime of excited state in gravity system:

$$
\tau_{\text {grav }}=\frac{K_{G}}{\Delta E} .
$$

Here $\Delta E$ is energy level width of an excited system determined by the energy removed by radiation of every type (neutrino, gamma, etc.). With Type I supernova outburst the radiated energy is equal to $\Delta E=10^{42}-10^{43} \mathrm{~J}$ [44] [45]. Then from relations (33) and (34) with $\mathrm{M}_{s}=1.99 \times 10^{30} \mathrm{~kg}, G_{N}=6.67 \times 10^{-11} \mathrm{~kg}^{-1} \cdot \mathrm{m}^{3} \cdot \mathrm{s}^{-2}$, we obtain that the excited state lifetimes of collapsing stars are within the range $\tau_{\text {grav }}=0.17-1.7 \mathrm{~s}$. The obtained values correspond to generally known astrophysical concepts about times of "cold" neutron star formations as a result of supernova outburst. With Type II supernova outburst the radiated energy is equal to $10^{41} \mathrm{~J}$ [45]. According to relations (33) and (34) this provides the lifetime of the star excited state $\tau_{\text {grav }}=17 \mathrm{~s}$. This value corresponds to astrophysical concepts about the time of cooling-down of a "hot" neutron star formed as a result of slow escape of supernova shell [45] which is equal in order of magnitude to $10 \mathrm{~s}$. It should be noted that the discovered time range of fast and slow neutron star formation 0.17 - $17 \mathrm{~s}$ covers the range (set in [24] [34]) of times of nucleosynthesis and forced fission yields and times of formation of final element abundance in Galaxy. Hence the time $\tau_{\text {rot }} \sim 10^{-20} \mathrm{~s}$ corresponds to formation of heavy and superheavy clusters inside composite nuclei, and the times $\tau_{\text {grav }}=0.17-17 \mathrm{~s}$ correspond to formation of recorded distribution of elements bulk formed during processes of gravity compression of supernova central regions.

We try to determine a size of the gravitating nuclear matter cluster (neutron star) on the basis of "momentumcoordinate" uncertainty relation, written for astrophysical objects:

$$
\Delta p \cdot \Delta a \cong M V a_{s} \geq \frac{K_{G}}{2} .
$$

Here, $M$ is object mass, $V$ is typical velocity of compression process, $a_{s}$ is object localization, i.e., size of star. From here we get the following expression for object localization:

$$
a_{s} \cong \frac{K_{G}}{2 M V} \cong \frac{\mathrm{M}_{C h}^{2} G_{N}}{2 M V C} .
$$

In case of relativistic collapse $(V \sim c)$ the object size is equal to $a_{s} \cong \mathrm{M}_{C h}^{2} G_{N} / 2 M c^{2}$. Since $M \sim \mathrm{M}_{C h}, a_{s}$ is lower than the gravitational radius $r_{g}=2 M G_{N} / c^{2}$, which means the formation of black hole. If a typical process velocity corresponds in order of magnitude velocity of supernova shell escape (about 20,000 km.s ${ }^{-1}$ [45]), then object localization is $1.56 \times 10^{4} \mathrm{~m}$. The obtained value corresponds to the minimum size of a typical neutron star equal to $16 \mathrm{~km}$ [44].

\section{Mesoscopic Objects}

Since the proposed kinetic approach provides an adequate description of processes in microcosm, it is possible to try to use it for considering object growth processes in mesoscopic scales. This section provides a data review are in view on development of the proposed asymptotic method for investigating "mesokinetics" of growth of various nanostructured objects with strong interatomic bonds [15]. Among these are carbon nanostructures, for example, single-wall nanotubes and detonation nanodiamonds [21] [47] (covalent bonds) as well as light actinides with strong approach of atoms [9] ("quasi-covalent" bonds). It is assumed that the growth of nanostructures is caused by their phonon excitations and vibrational interactions, leading to mutual compensation of free electronic bonds of "boundary" (i.e., surface) atoms in adjoining objects and to consolidation of these objects into more large patterns, which are compact clusters.

At the initial stage of crystal objects growth, as a result of random interactions between seeds carrying crystal 
structure elements, small crystalline particles having the short-range-order are formed. These small particles continue to interact with seeds and between themselves, causing their growth. In the process of this growth the quasi-long range crystal ordering arises in the particle, and the lower limit of complexity for a macroscopic solid is reached. Hence the growth process of mesoobjects with nanocrystalline structure can be considered as formation of clusters in continuum initially consisting of seeds with the set spatial orientation of atoms [15] [25].

With phenomenological description of single-wall carbon nanotube growth as a result of capture of hexagon seeds based on uncertainty relation (1) the following formula was obtained for estimating a typical nanotube diameter [48]:

$$
d=\frac{3 \sqrt{3} l_{\mathrm{cov}}^{2}}{2 \pi \hbar} \sqrt{\frac{A m_{u} k_{B} \theta_{v}}{\pi}}
$$

where $\theta_{\mathrm{v}}$ is characteristic oscillation temperature of covalent bond tension in C-C carbon atoms, $l_{\text {cov }}$ is carbon covalent bond length ( $m_{u}$ is atomic mass unit, $k_{B}$ is the Boltzmann constant). It follows here from that a typical diameter of a single-wall carbon tube is $d=2.2 \mathrm{~nm}\left(\theta_{\mathrm{v}}=2154 \mathrm{~K}, l_{\text {cov }}=0.15 \mathrm{~nm}\right)$. This value is in the middle of experimental range $d=1-4 \mathrm{~nm}$ given in [21]. The expression obtained in [48] for the growth of an average length of a single-wall carbon nanotube looks like:

$$
\langle l\rangle=\left(\frac{\hbar t}{2 m_{0}}\right)^{1 / 2}
$$

The parameter $\hbar / 2 m_{0}$, by analogy with quantum mechanics [49], can be determined as the upper limit of a diffusion coefficient in the space of cluster sizes. The expression obtained for $\langle l\rangle$ represents a quantum analog of a classical diffusion solution [50] of a problem about growth of particle of new phase and specifies the least possible time of growth in the system initially consisted of seeds (hexagons). From expression (37) we obtain that the least growth time for a single-wall carbon nanotube with the given in [21] length of $40 \mathrm{~nm}$ is $4 \mu \mathrm{s}$. It should be noted that in this case we are dealing with the time of growth for an individual nanotube, not with hardware time of synthesis of a bulk of these objects. Of some certain interest is the condition determination for performance of experiments, when single-wall nanotubes with the given average size specified according to expression (37) will grow as a result of phonon effects.

When considering formation and growth of volume-packed objects at average temperatures compared to Debye temperature, we will distinguish between "small" clusters in which atom behavior corresponds to oscillation spectrum of seeds not having a crystalline structure, and "large" clusters in which behavior of atoms corresponds to dynamics of crystal lattice oscillations. A crystal lattice can have either the short-range-order or quasilong-range ordering depended on a cluster size. At the initial stage in processes of collisions between seeds the "first" clusters are formed. As a result of interactions between these clusters and seeds the larger objects are formed in the system. A typical time scale for them can be evaluated as a product of a number of bonded seeds in an object equal by order of magnitude to $\left(\langle a\rangle / a_{0}\right)^{3}$ and the time $t_{\text {exc }}$ of excitation of a single seed:

$$
t_{i} \approx\left(\frac{\langle a\rangle}{a_{0}}\right)^{3} t_{e x c} .
$$

The parameter $t_{\text {exc }}$ can be determined as a product of number $N$ of chemical bonds in a seed and the inverse typical frequency $v_{v}^{-1}$ of atom oscillations in its structure, namely, $t_{\text {exc }}=N v_{v}^{-1}$. Characteristic oscillation frequency can be expressed through the relevant characteristic temperature $\theta_{v}$ based on the relation $2 \pi \hbar v_{v}=k_{B} \theta_{v}$. Then in compliance with the expressions (8) and (9) the approximate law of growth for an average size in processes of interaction between small clusters and seeds and between them will be written the following way:

$$
\langle a\rangle \cong a_{0} 2^{1 / 4}\left(\frac{t}{t_{u n}^{s c}}\right)^{1 / 2}, \quad t_{u n}^{s c}=a_{0}\left(\frac{m_{0} t_{e x c}}{\hbar}\right)^{1 / 2} .
$$

Here, $t_{u n}^{s c}$ is a time unit in interaction processes between small clusters. By classical analogy with notions [50] the exponent 1/2 in the Formula (38) corresponds to the diffusion of seeds at the cluster. 
Clusters of size progressively increasing in time are formed in the system. They can interact with both seeds and between themselves as well. The minimum object size $\langle a\rangle_{\min }$ at which an oscillating spectrum of a small cluster switches over an oscillating spectrum of a large cluster "by a jump", corresponds to the occurrence of the crystal short-range ordering, and represents the lower boundary of transition region from mesoscopic structures to macroscopic bodies. As crystal structures grow, they tend to the quasi-long-range ordering, and the dynamic system itself "forgets" about initial conditions. It would appear natural that at a certain time some critical average cluster size $\langle a\rangle_{*}$ is reached in this system. This size does not depend on a seed size and is determined only by dynamics of atom oscillations in investigated objects. This corresponds to the quasi-long-range crystal ordering emerged in the system, i.e., to the lower limit of complexity reached in a macroscopic crystal body. The given size corresponds to the upper boundary of a transition region from mesoscopic structures to macroscopic bodies.

The characteristic size $\langle a\rangle_{\min }$ of objects, beginning with which the cluster has got the crystal lattice with short-range order, and the characteristic size $\langle a\rangle_{*}$, such that quasi-long-range crystal ordering arises in the cluster, and the appropriate times of achievement of these sizes were obtained in [15] [25] on the basis of the laws (38), (22, 23) as follows:

$$
\begin{gathered}
\langle a\rangle_{\min } \cong\left(\frac{9}{8}\left(\frac{4 \pi}{3}\right)^{1 / 3} a_{0} \frac{N}{n^{1 / 3}} \frac{\theta_{D}}{\vartheta_{v}}\right)^{1 / 2} \\
\langle a\rangle_{*} \cong \frac{18}{25}\left(\frac{4 \pi}{3}\right)^{1 / 3} n^{-1 / 3} \exp \frac{E_{v}-E_{0}}{2 k T} \\
t_{\min } \cong \frac{9}{8 \sqrt{2}}\left(\frac{m_{0} c_{0}^{2} t_{\text {exc }}^{3}}{\hbar}\right)^{1 / 2} \\
t_{*} \cong \frac{16}{5}\left(\frac{3}{5}\right)^{5}\left(\frac{m_{0} c_{0}^{5} t_{s}^{6}}{\hbar a_{0}^{3}}\right)^{1 / 2}
\end{gathered}
$$

Here, $\theta_{D}$ is Debye parameter, $n$ is concentration of atoms in a crystal lattice, $t_{s}=\left(2 \pi \hbar / k_{B} \theta_{D}\right) \exp \left[\left(E_{v}-E_{0}\right) / 2 k_{B} T\right]$, $E_{0}$ is seed energy in the environment determined by equations for inner energy of nonlinear polyatomic molecules given in [51]; $E_{v}$ is seed energy inside a large cluster determined by an oscillating spectrum of a small particle [52]:

$$
E_{0}=3 k T+k T\left\{\sum_{v=1}^{N} \frac{\theta_{v}^{(1)} / T}{\left\{\exp \left[\theta_{v}^{(1)} / T\right]\right\}-1}+\frac{(3 M-6)-N}{N} \sum_{v=1}^{N} \frac{\theta_{v}^{(2)} / T}{\left\{\exp \left[\theta_{v}^{(2)} / T\right]\right\}-1}\right\}
$$

$\theta_{v}^{(j)}(j=1,2)$ are typical temperatures of tension vibrations and flexural vibrations of atom bonds in a molecule, respectively; $M$ is number of atoms in a molecule, $N$ is number of bonds:

$$
E_{V}=M\left\{|\chi|+3 D k T\left(T / \theta_{D}\right)^{D} \int_{0}^{\theta_{D} / T}\left[x^{D} /(\exp x-1)\right] \mathrm{d} x\right\}, x=2 \pi \hbar v / k T
$$

Here, $|\chi| \cong k \theta_{D}$ is energy of the basic lattice oscillating state (energy of "zero" oscillations), $D$ is internal (dynamic) fractal dimensionality of cluster, $v$ is oscillation frequency of atoms in the lattice. Formulae (39), (40) determine the area of average cluster sizes $\langle a\rangle \in\left\{\langle a\rangle_{\min } \ldots\langle a\rangle_{*}\right\}$ which is a transition region from mesoscopic structures to macroscopic particles.

The growth laws for crystal particles from seeds (polyatomic molecules) look like [15] [53]:

$$
\begin{gathered}
\langle a\rangle \cong\left(\frac{25 k \theta_{D} a_{0}^{3}}{\left.16 A M m_{u} \exp \frac{E_{V}-E_{0}}{2 k T}\right)^{1 / 5} t^{2 / 5}}\right. \\
\langle a\rangle \cong\left(\frac{9 k \theta_{D} a_{0}^{3}}{4\left(6 \pi^{2} n\right)^{1 / 3} A M m_{u}}\right)^{1 / 6} t^{1 / 3}
\end{gathered}
$$


Here, $M$ is number of atoms in a seed. Formula (43) describes growth of large clusters under small flux of seeds, Formula (44) corresponds to both large flux of seeds and interaction between large clusters, sound speed being recorded in Debye approximation as $c_{0} \cong k \theta_{D} / \hbar\left(6 \pi^{2} n\right)^{1 / 3}$.

In case of detonation synthesis of nanodiamonds, a "Lagrangian" layer of shock compressed confined matter (i.e., a layer with a constant mass) is viewed as a closed system [53]. It is assumed that deformation and fragmentation of benzene rings are taking place in a shock wave front that results in production of structural seeds of diamond phase with cubic modification-molecules of cyclohexane carbon skeleton. An effective size of such a seed is determined by the length of a covalent bond of carbon atoms and is equal to $a_{0}=0.3 \mathrm{~nm}$. The estimated time of seed formation $t_{n f} \sim 10^{-12} \mathrm{~s}$ was obtained in [53] from kinetic considerations. As shock compressed layer expands, crystal diamond-like clusters are formed in it as a result of seed coalescence and oscillating interactions between evolving nanostructures and seeds and between themselves.

The minimum average size of diamond-like nanoclusters starting from which they possess a crystal lattice, is estimated as $\langle a\rangle_{\min } \sim 10^{-9} \mathrm{~m}$. The cluster of this size has $\left(\langle a\rangle_{\min } / a_{0}\right)^{3} \cong 3^{3}$ seeds, i.e. about 160 carbon atoms. In accordance with Formula (41) the formation time of such cluster can be estimated as $t_{\min } \sim 10^{-12} \mathrm{~s}$. Using Formula (40) and the time of seed capture determined in [54] as $t_{s}=3 \times 10^{-13} \mathrm{~s}$ one can estimate the cluster minimum size starting from which it can be regarded as a macroscopic particle: $\langle a\rangle_{*} \sim 2 \times 10^{-9} \mathrm{~m}$. This cluster includes $\left(\langle a\rangle_{*} / a_{0}\right)^{3} \cong 7^{3}$ seeds, i.e., approximately 2000 carbon atoms. From Formula (42) we obtain that the time of synthesizing of this particle is equal to $t_{*} \sim 4 \times 10^{-10} \mathrm{~s}$. The further growth of diamond-like particles is described by Formulae (43) and (44). Estimates provided in [53] [54] show that an average size of detonation nanodiamonds about $4 \mathrm{~nm}$ can be reached in a time of about $(2-5) \times 10^{-9}$ s. These estimates as well as asymptotic mode (4) of cluster size distribution and the idea about existence of local maximum variety within this distribution agree with key experimental data obtained by different authors (see [25]).

Shock-induced coalescence of detonation nanodiamonds of "water" synthesis under dynamic loading in recoverable ampoule is described in [53] [55]-[57]. Growth of particles is described by the following formulae obtained using the above laws (22), (23):

$$
\begin{gathered}
\langle a\rangle \cong\left(\frac{75 k \theta_{D}}{8 \pi A m_{u} n}\right)^{1 / 5} t^{2 / 5} \\
\langle a\rangle \cong\left(\frac{27 k \theta_{D}}{2 \times 6^{1 / 3} \pi^{5 / 3} A m_{u} n^{4 / 3}}\right)^{1 / 6} t^{1 / 3} .
\end{gathered}
$$

Formula (45) corresponds to a small flux of seeds (nanodiamonds), when an average size growth occurs as a result of "particle-seed"- type interaction. Formula (46) corresponds to a large flux of seeds, when growth occurs as a result of "particle-particle"-type interaction. Because the compression pulse duration is short $\left(\tau \sim 10^{-5}\right.$ s), it is natural to assume that the crystal particles predominantly grow under metastable conditions for the diamond in the phase diagram of carbon: at zero pressure and increased residual temperatures. Cooling-down time for shock heated porous carbon sample is determined using the solution of problem about a heat wave starting from an instant plane source (a thin disk) formed under shock compression of nanodiamond powder and propagating of this wave in material of the ampoule. Estimates performed according to Formulae (45), (46) show that during a cooling-down time of about $t \sim 10^{2} \mathrm{~s}$ two subsystems of crystal diamond-like particles with average sizes about $1.5 \times 10^{-5} \mathrm{~m}$ and $1.5 \times 10^{-4} \mathrm{~m}$ are formed in porous sample of nanodiamonds. The mechanism of crystal growth resulted from consecutive connection of "small" seeds to a "large" particle described by Formula (45) provides by an order of magnitude larger sizes than the mechanism of large particle coalescence described by Formula (46). The calculated results agree with the given in [57] experimental bimodal distributions of polycrystalline diamond particles under consideration.

Behavior of light actinides in conditions of internal self-irradiation and external effects in many cases is determined by their phase stability and a character of crystal phase polymorphic transformations [9]. The problem of phase stability and polymorphic transformations of light actinides is considered in terms of phenomenological 
concepts about evolution of nanoclusters as a result of their phonon excitation and oscillating interactions [15]. The basis for such concepts is, for example, the existence of strong (quasi-covalent) bonds in multiphase plutonium resulted from $f$-electrons effect on atoms approaching each other [9].

First, we will single out the following process: a) spontaneous formation of plutonium alpha-phase $(\alpha-\mathrm{Pu})$ from plutonium delta-phase ( $\delta$-Pu) as a result of sample aging; b) martensitic transition of plutonium delta-phase into alpha-prime $\left(\alpha^{\prime}\right)$ phase on cooling of samples and reverse transformation on their subsequent heating. We will suppose that at the initial time there is a fairly large number of starting seeds of a certain crystalline phase which could be generated, as a result of temperature fluctuations, on defects of the initial structure either affected by intrinsic radioactive radiation, or as a result of local shear deformations on lattice defects under external action. Since the $\alpha^{\prime}$ phase of Pu differs from the $\alpha$-phase by the presence of a relatively small number of gallium (Ga) impurity atoms ( 1\% - 2\%) and crystal lattice parameters of these phases are close to each other (see, for example, [9]), we are going to consider the «pure» transitions $\delta \rightarrow \alpha \rightarrow \delta$ for simplicity.

Therefore, the influence of impurity atoms on kinetics of phase transformations is ignored in a proposed model. As starting seeds of $\alpha$-Pu we will consider nanoclusters consisting of eight atoms with the only crystallographically possible mutual orientation described in [9]. A characteristic size of an $\alpha$-phase seed, accepted to be equal to the most linear size of an elementary crystal cell, is $a_{0 \alpha}=1 \mathrm{~nm}$; the time of its formation from dimmers of $\delta$-phase is evaluated as:

$$
t_{n \alpha}=\frac{2 \pi \hbar}{k_{B} \vartheta_{v}} \exp \frac{\varepsilon_{\alpha}-4 \varepsilon_{\delta}}{k_{B} T} \sim 10^{-12} \mathrm{~s}
$$

Here, $\varepsilon_{\alpha}$ is internal energy of $\alpha$-phase seed, and $\varepsilon_{\delta}$ is internal energy of the dimer. Due to connection of two such clusters a unit cell is generated which consists of 16 atoms, and which forms $\alpha$-Pu monoclinic lattice. Dimmers, tetrahedrons, as well as plane formations of six atoms generating glide planes of face-centered cubic lattice (fcc) can be considered as structural seeds of $\delta$-Pu. Irreversible aggregation of seeds results in formation of small (nano-) particles with quasi-crystal (short-range) ordering of atoms in a lattice. Estimates show that the minimum size and time for forming a particle with a short-range ordering in $\alpha$-Pu crystal lattice are equal to $\langle a\rangle_{\min } \sim 2 \times 10^{-9} \mathrm{~m}, t_{\min } \sim 1.9 \times 10^{-10} \mathrm{~s}$, respectively. Estimated parameters determine premartensitic state described in [9]. The following coalescence of such nanocrystal objects brings to formation of larger particles with quasi-long range ordering.

Based on uncertainty relation (1') and the resultant expression (46) in Debye approximation for description of nanoparticle coalescence in [58], it was stated that at modest temperatures the spontaneous generation of $\alpha$-Pu polycrystals with sizes of about $10^{-7} \mathrm{~m}$ occurs for the time $t=6 \times 10^{-3} \mathrm{~s}$. Formally it was also obtained that $\alpha$-Pu "macroscopic" particles with sizes $0.1-1 \mathrm{~mm}$ can be generated for times of $2 \times 10^{-1}-2 \times 10^{2}$ years. It should be noted that these estimates are in compliance with the generally known concepts about slow kinetics of spontaneous transformation of alloyed $\delta$-Pu in normal storing conditions.

The main mechanism of martensitic transition in the proposed model is addition of starting seeds (polyatomic molecules) to large clusters in the regime of small flux of seeds, when each of them has time to occupy its energy advantageous place on a surface of a large cluster before the interaction with the following seed begins. In this case growth of a martensite particle is described by Formula (22) in which the process finish time, according to concepts [59] about ordering kinetics at phase transformations, is determined as:

$$
t_{\text {end }}=\frac{l}{c_{\delta}} \exp \frac{E_{v}-E_{0}}{k_{B} T}
$$

Here, $l$ is a characteristic size of an initial matrix from $\delta$-Pu, e.g., a grain average size in a homogenized sample, $c_{\delta}$ is velocity of propagation of minor elastic perturbations in the initial matrix. A limited average size of particles formed as a result of capturing the seeds with oscillating spectrum of polyatomic molecule [60] automatically follows from (22), (43), (47):

$$
\left\langle a_{\alpha}\right\rangle_{\lim } \cong\left(\frac{25 k_{B} \theta_{D \alpha} a_{0}^{3}}{16 A M m_{u} \exp \frac{E_{v}-E_{0}}{2 k_{B} T}}\right)^{1 / 5}\left(\frac{l}{c_{\delta}} \exp \frac{E_{v}-E_{0}}{k_{B} T}\right)^{2 / 5} .
$$


Here, $\theta_{D \alpha}$. is Debye parameter of $\alpha$-phase. Temperature $T_{0}$ of the beginning of martensitic transformation is determined from the condition that an average $\alpha$-phase particle size becomes equal to a characteristic longitudinal size $l_{\text {long }}$ of a twin. If this value does not depend on $T$, then it follows from Formula (48) that with the decreased $l$, the value $T_{0}$ has to decrease as well, since in oscillating spectrum $E_{v}$ of solid particles there is a temperature-independent term connected with zero oscillations of a crystal lattice [15] [52]. Finish temperature $T_{*}$ of martensitic transition is determined from the condition that average particle size of $\alpha$-phase becomes equal to a critical size at which a quasi-long-range ordering appears in the system, that is from the condition of intersection of the curve $\langle a\rangle_{*}=f(T)$ (geometric place of points in a plane $(\langle a\rangle, T)$ determined according to Formula (40)) with the curve in the plane $(\langle a\rangle, T)$ by Formula (48): $\left\langle a_{\alpha}\right\rangle_{\text {lim }}=\langle a\rangle_{*}$. In this case the condition $t_{\text {end }}=t_{*}$ is fulfilled automatically. With lower temperatures $T<T_{*}$, when $t_{\text {end }}<t_{*},\left\langle a_{\alpha}\right\rangle_{\lim }<\langle a\rangle_{*}$, the quasi-long-range ordering does not have time to be formed, and the system does not reveal explicit macroscopic properties. From the condition $\left\langle a_{\alpha}\right\rangle_{\lim }=\langle a\rangle_{*}$ we obtain the following equation to determine a "critical" temperature of martensitic transition finish:

$$
\frac{E_{v}-E_{0}}{k_{B} T_{*}}=\ln \left\{\left(\frac{25 k_{B} \theta_{D \alpha} a_{0}^{3}}{16 A m_{u}}\right) \frac{n_{\alpha}^{5 / 3}}{\left.\frac{18}{25}\left(\frac{4 \pi}{3}\right)^{1 / 3}\right]^{5}}\left(\frac{l}{c_{\delta}}\right)^{2}\right\} .
$$

From here, it follows that with the decreased $l$, temperature $T_{*}$ should increase through the presence (in the expression for $E_{v}$ ) of the term responsible for zero oscillations and independent on temperature.

The calculated curves $T(l)$ given in [25] with characteristic temperatures of martensitic transition show that in a diffusion model [60], with the decreased matrix grain size, transformation "blurring" determined as the difference $T_{0}-T_{*}$, decreases also, which is caused by the effect of crystal lattice zero oscillations under significant sample.

Now, we consider the reverse transformation $\alpha \rightarrow \delta$ during the heating of the sample preliminary cooled to the "critical" temperature $T_{*}$. In this case, the vibration spectrum of the small $\alpha$-Pu particle surrounded by the $\delta$-Pu matrix transforms into the vibration spectrum of the environment due to the interaction of atoms at the interface. This process propagates deep into the particle and leads to the transformation of its vibration spectrum in the bulk; i.e., the polymorphic transformation occurs. According to the differential relation (25) when $\mathrm{N}=n\langle a\rangle^{3}$, we can obtain the following relationship for the growth of the reverse transformation region during the heating over the entire volume:

$$
\left\langle a_{\delta}\right\rangle \cong \frac{\sqrt{2}}{2}\left(\frac{3 k_{B} T D(x)}{A m_{u}}\right)^{1 / 2} t .
$$

Here, $D(x)$ is the tabulated Debye function with $x=\theta_{D} / T$ [61]. Formula (49) determines the lower time boundary of the inverse transformation which is evaluated in [15] [60] as $t_{\min } \sim 10^{-8} \mathrm{~s}$.

As the size of inverse transformation region increases, which is described by Formula (49), this region can capture some individual groups of atoms (atomic clusters) from the initial matrix surface from the $\delta$-phase in the region of "contact" with $\alpha$-phase and recovered $\delta$-phase. In addition clusters can be captured from $\alpha$-phase martensitic particles. The inner energy of clusters can correspond to either intermediate state between two crystal phases (diatomic and polyatomic molecules), or atom oscillation spectrum in a solid. The law of growth of reverse transformation region in the mode of a small flux of seeds described by Formula (43) looks like:

$$
\left\langle a_{\delta}\right\rangle \cong\left(\frac{75 k_{B} \theta_{0 \delta}\left(1-A_{\delta} T\right) a_{0}^{3}}{16 \pi A M m_{u} \exp \left(\left(E_{v \delta}-E_{0 i}\right) / 2 k_{B} T\right)}\right)^{1 / 5} t^{2 / 5}
$$

Here, $E_{0 i}$ is the energy of the captured object determined by a number of atoms in a cluster, index $i$ is phase, $E_{v \delta}$ is energy of the captured object inside the region of inverse transformation, determined by an oscillating spectrum of a solid particle. It is taken into consideration here that as a result of elasticity here, we took into account that the elasticity softening leads to a decrease in the Debye parameter according to the linear law 
$\theta_{D i}=\theta_{0 i}\left(1-A_{i} T\right), \theta_{0 i}$ is Debye parameter at $T=0$ [62]. Formula (50) determines the upper time boundary for the inverse transformation within the proposed model estimated as $2.4 \mathrm{~s}$ [15] [60].

In case of capturing seeds directly from $\alpha$-phase, when their vibration spectrum $E_{v \alpha}$ corresponds to the solid body vibration spectrum, expression (43) for an average size of $\delta$-phase particles looks like:

$$
\left\langle a_{\delta}\right\rangle \cong\left(\frac{75 k_{B} a_{0}^{3} \theta_{0 \delta}\left(1-A_{\delta} T\right)}{16 \pi A M m_{u} \exp \left(\left(E_{v \delta}-E_{v \alpha}\right) / 2 k_{B} T\right)}\right)^{1 / 5} t^{2 / 5}
$$

Within the temperature range of the reverse transformation $\alpha \rightarrow \delta$ the linear dependence [62] for Debye parameter can be approximately written as $\theta_{D \delta} \approx \theta_{0 \delta} \exp \left(-A_{\delta} T\right)$. In case of dimmers as $\delta$-phase seeds, the internal cluster energy is written as:

$$
E_{0 i}=k_{B} T\left(\frac{5}{2}+\frac{\vartheta_{v i} / T}{\exp \vartheta_{v i} / T-1}\right) \approx k_{B} T\left(\frac{5}{2}+\left(1-\frac{1}{2} \frac{\vartheta_{v i}}{T}\right)\right),
$$

where the first two expansion terms (using Bernoulli numbers $B_{n}$ [63]) are taken into consideration: $x /\left(\mathrm{e}^{x}-1\right) \approx 1-(x / 2)+\left(B_{1} x^{2} / 2 !\right)-\left(B_{2} x^{4} / 4 !\right)+\cdots, \quad x^{2} \ll 4 \pi^{2}, B_{1}=1 / 6, B_{2}=1 / 30, \cdots$. If one neglects terms of the second (or higher) order of smallness, the temperature factor in Formula (50) in the first approximation can be written as:

$$
F(T) \approx \exp \frac{1}{5}\left[-A_{\delta} T-\frac{7}{4}-\frac{1}{4} \frac{\vartheta_{v i}}{T}\right] .
$$

From the condition of extremeness:

$$
F^{\prime}(T)=\left(-A_{\delta}+\frac{1}{4} \frac{\vartheta_{v i}}{T^{2}}\right) \exp \frac{1}{5}\left[-A_{\delta} T-\frac{5}{4}-\frac{1}{4} \frac{\vartheta_{v i}}{T}\right]=0
$$

we obtain the following expression for determining the "relevant" temperature:

$$
T_{i}=\sqrt{\frac{1}{4} \frac{\vartheta_{v i}}{A_{\delta}}}
$$

At this point the second derivative is negative: $F^{\prime \prime}(T)<0$. The values $T_{\mathrm{i}}$ determine temperatures at which particle sizes growing from dimers of this or that phase reach their maximum in a plane $(\langle a\rangle, T)$. In case of $\delta$-phase dimers the $T_{i}$ value corresponds to the temperature $T_{s}$ of the beginning of the reverse transformation $\alpha \rightarrow \delta$ :

$$
T_{s}=\frac{1}{2}\left(\frac{\vartheta_{v \delta}}{A_{\delta}}\right)^{1 / 2} .
$$

In case of $\alpha$-phase dimmers, the $T_{i}$ value corresponds to the temperature $T_{f}$ of the reverse transformation $\alpha \rightarrow \delta$ finish:

$$
T_{f}=\frac{1}{2}\left(\frac{\vartheta_{v \alpha}}{A_{\delta}}\right)^{1 / 2}
$$

Knowing the critical temperature $T_{*}$ of martensitic transition finish and the temperature $T_{s}$ of the reverse transformation beginning at heating one can determine the temperature hysteresis $T_{s}-T_{*}$.

A temperature factor in Formula (51) for an average size of the reverse transformation region is written as:

$$
F(T) \cong \exp \frac{1}{5}\left[-A_{\delta} T+\frac{3 M}{2} \frac{\theta_{0 \alpha}^{2}-\theta_{0 \delta}^{2}}{20 T^{2}}\right] .
$$

It is taken into consideration that at high temperatures $T \gg \theta_{D}$ the vibration energy in a crystal lattice per an atom is equal to (see [51])

$$
E_{v i} / M \approx 3 k_{B} T\left[1+\frac{1}{20}\left(\frac{\theta_{D i}}{T}\right)^{2}\right] .
$$


In the plane $(F, T)$ there exists a special point, in which exponent index is equal to zero, and $F(T)=1$, i.e., elastic vibrations of atoms are balanced by elasticity softening. With higher temperatures elasticity softening becomes predominant and inverse transformation "decelerates". From here we obtain the following expression for the value $T_{M}$ corresponding to the decrease of average size growth rate with temperature for the given number of atoms $M$ in cluster seed with vibrational spectrum of the solid:

$$
T_{M}=\left(\frac{3 M}{2} \frac{\theta_{0 \alpha}^{2}-\theta_{0 \delta}^{2}}{20 A_{\delta}}\right)^{1 / 3}
$$

It can be seen from relationship (54) that there exists a discrete spectrum of characteristic temperatures $T_{M}$ each of which corresponds to a particular number $M$. This discrete spectrum determines a stepwise character of particle growth during the heating due to the capture of clusters with different numbers of atoms.

Discrepancy in calculated and experimental data [64] relating to characteristic temperatures of the martensitic transition beginning and finish and of the reverse transformation is 2\% - 24\% [60]. The calculated, using Formula (54), discrete set of temperatures $T_{M}=318,335,350,364 \mathrm{~K}$ at which rate of growth of a particle average size with atom numbers in the seed $M$ from 6 to 9 decreases, corresponds to the temperature interval [65] 328 $360 \mathrm{~K}$. Within this interval one can observe "steps" on dilatometric curves for relative extension of samples and the corresponding "bursts" on curves for velocities of sample extension in experiments [65].

The established correspondence gives foundation for determining the least grain size of the $\delta$-phase initial matrix at which martensitic transformation into $\alpha$-phase is still possible. As a necessary condition for martensitic transformation one can accept that an average size of a martensite particle should be no less than a characteristic longitudinal size of a twin $l_{\text {long: }}\left\langle a_{\alpha}\right\rangle_{\text {lim }} \geq l_{\text {long }}$. The proper equation for the minimum grain size of $\delta$-phase matrix lower which the martensitic transformation does not occur, since a typical twin size is not reached at cooling down to reasonably low temperatures about $100 \mathrm{~K}$ obtained from (48), looks like:

$$
l_{\min } \cong l_{\text {long }}\left(\frac{16 A m_{u} c_{\delta}^{2}}{25 k_{B} \theta_{D \alpha} a_{0}^{3} n_{\alpha}}\right)^{1 / 2}\left[\frac{18}{25}\left(\frac{4 \pi}{3}\right)^{1 / 3}\right]^{3 / 2} .
$$

Hence at $l_{\text {long }}=1 \mu \mathrm{m}$ [9] we obtain $l_{\min }=2.2 \mu \mathrm{m}$. This grain size is in compliance with the calculated temperatures of the beginning $T_{0}$ and finish $T_{*}$ of martensitic transition equal to 140 and $110 \mathrm{~K}$, respectively. Values $l_{\text {min }}$ $=2.2 \mu \mathrm{m}, T_{0}=140 \mathrm{~K}$ and $T_{*}=110 \mathrm{~K}$ can be considered as limiting for this mechanism of martensitic

particle growth. From Formula (48) it follows that $\left\langle a_{\alpha}\right\rangle_{\text {lim }} \approx 0.45 l$. This means that the grain size less than $l_{\text {min }}=$ $2.2 \mu \mathrm{m}$ can be reached as a result of thermal cycling, i.e., with sequential cycles of "cooling down to $\alpha$-phase transition $\rightarrow$ heating back up to $\delta$-phase $\rightarrow$ the following cooling down to $\alpha$-phase transition” and so on. Since with $\delta$-phase recovery a typical matrix grain size decreases to the value $0.45 l$, with the following cooling, the martensitic particle size will be equal to $0.2025 \mathrm{l}$, hence at heating, a typical grain size of $\delta$-Pu matrix will be equal to $0.2025 \mathrm{l}$ and etc. in decreasing geometric progression. With the given initial size $l$, it is possible to obtain in just several cycles such size of $\delta$-phase matrix grain that will be lower than $l_{\min }$. The fact that the size of $\alpha$-phase martensitic particle is always less than that of $\delta$-phase initial matrix grain means that the martensite total volume at the following cycles will be always less than the martensite total volume at the previous phase. And at each further cycle the temperature of the martensitic transition beginning will be lower than that at the previous cycle. The results are in qualitative agreement with the experimental data [66] relating to $\delta$-Pu stabilization at thermal cycling.

Under long annealing of samples the homogenizing and increase of their grain sizes occur after each cycle that brings to martensite transformations in their former volume. Sample homogenizing can take place as a result of coalescence of particles undergone the $\alpha \rightarrow \delta$ inverse transformation. With a point contact of such particles the parameter $\mathrm{N}$ in Equation (25) is equal to number of atoms in a channel formed by linear chain of elementary crystal cells arranged within the length $2\langle a\rangle$ : where $N_{\mathrm{c}}$ is a number of atoms in a cell, and $a_{\mathrm{c}}$ is a typical cell size. Then from (25) we obtain the following equation for the growth of average size of the homogenized region $\langle a\rangle$ :

$$
\langle a\rangle \cong\left(\frac{12 N_{c} k_{B} T}{a_{c} A m_{u} n}\right)^{1 / 4} t^{1 / 2}
$$

Estimates in accordance with this formula show that with the temperature $T=600 \mathrm{~K}$ at the time $t=30 \mathrm{~min}$ the 
size of the region of homogenization reaches a value of about $2 \mathrm{~cm}$. Exactly this explanation can be applied to the data [67] on plutonium sample annealing.

In [15] the nature of $\delta$ - $\mathrm{Pu} \rightarrow \alpha$-Pu transition with the sample cooling down to the lower temperatures than those used in [64] is determined. In this case an $\alpha$-phase particle with the minimum size $\langle a\rangle_{\min }=2 \mathrm{~nm}$ (the particle having a short-range crystal ordering) can take out of the surface of $\delta$-phase surrounding matrix groups of atoms (clusters) with the solid body vibration spectrum. In the course of the interaction of the cluster with the particle the vibration spectrum of the cluster is transformed into the vibration spectrum of the $\alpha$-phase.

With temperatures $T \ll \theta_{D}$ the Equation (48) for the limiting average size of an $\alpha$-phase particle is written as:

$$
\left\langle a_{\alpha}\right\rangle_{\lim } \cong\left(\frac{25 k_{B} \theta_{D \alpha} a_{0}^{3}}{16 A M m_{u}}\right)^{1 / 5}\left(\frac{l}{c_{i}}\right)^{2 / 5} \exp \frac{3}{5} \frac{M}{2}\left[\frac{9}{8} \frac{\theta_{D \alpha}-\theta_{D \delta}}{T}+\frac{3 \pi^{4}}{5}\left(\frac{T^{3}}{\theta_{D \alpha}^{3}}-\frac{T^{3}}{\theta_{D \delta}^{3}}\right)\right]
$$

Here, it is taken into consideration that in Debye approximation [51] at minor temperatures the "specific" internal energy of a solid per an atom is equal to:

$$
\frac{E}{M}=\frac{9}{8} k_{B} \theta_{D}+3 k_{B} T \frac{\pi^{4}}{5} \frac{T^{3}}{\theta_{D}^{3}}, \frac{T}{\theta_{D}} \ll 1
$$

The value $\left\langle a_{\alpha}\right\rangle_{\lim }$ basically can reach a typical size of $\delta$-phase initial matrix (for example, the size of a homogenized grain), i.e., full transition from $\delta$-phase into $\alpha$-phase occurs. The temperature $T_{\mathrm{ft}}$ of full $\delta \rightarrow \alpha$ transformation is determined from the condition $\left\langle a_{\alpha}\right\rangle_{\text {lim }}=l$. At very low temperatures, when in previous equations one can neglects the terms proportional to $T^{3}$, we obtain the following approximated equation for the temperature of full transformation:

$$
T_{f t} \cong \frac{3 M}{4} \frac{9}{8} \frac{\theta_{D \alpha}-\theta_{D \delta}}{\ln \left[\frac{16}{25}\left(\frac{l}{a_{0}}\right)^{3} \frac{A M m_{u} c_{\delta}^{2}}{k_{B} \theta_{D \alpha}}\right]}
$$

In accordance with Formula (47), the time when a martensitic particle achieves the size $l$, can be written as:

$$
\tau_{l}=\frac{l}{c_{\delta}} \exp \frac{3}{5} \frac{M}{2}\left[\frac{9}{8} \frac{\theta_{D \alpha}-\theta_{D \delta}}{T_{f t}}\right]
$$

Hence we obtain that with the grain size $\delta$-Pu $l=30 \mu \mathrm{m}$ the temperature of full $\delta \rightarrow \alpha$ transformation is $T_{f t}=$ $13.5 \mathrm{~K}$, and the time of this transformation is about $9 \times 10^{-2} \mathrm{~s}$. If as a result of preliminary thermocycling with moderate cooling the $\delta$-Pu grain size is decreased to the critical value $l_{\min }=2.2 \mu \mathrm{m}$, then the temperature of $\delta \rightarrow \alpha$ full transformation is $T_{f t}=17 \mathrm{~K}$, and the corresponding time is about $1.3 \times 10^{-3} \mathrm{~s}$. With the given temperatures critical sizes of $\alpha$-phase particles $\left\langle a_{a}\right\rangle_{*}$ estimated according to Formula (40) significantly exceed the sizes of initial $\delta$-phase grains. For example, at $T_{f t}=13.5 \mathrm{~K}$ we have $\left\langle a_{a}\right\rangle_{*}=220 \mu \mathrm{m}$ which is significantly higher than the initial grain size $l=30 \mu \mathrm{m}$. This means that in the $\alpha$-phase particle the long-range ordering is not reached. Such particle does not have macroscopic properties and represents a mesostructure with a short-range crystal ordering. At the same time sizes of such object are large as compared to those of a martensitic particle formed at cooling down to reasonably low temperatures about $100 \mathrm{~K}$, when $\left\langle a_{\alpha}\right\rangle<l$.

An important issue is the one relating to growing of macroscopic size plutonium crystals for investigation of their mechanical properties [67]. In the proposed models one can use as seeds nanoparticles with a critical size $a_{*}$, at which a particle has quasi-long-range crystal ordering. With small seed flux, when each seed has time to occupy its energy advantageous place on a cluster surface before it interacts with the following nanoparticle, the size of a phonon excitation region can be approximately equal to $a_{*}$. Then a number of excited atoms is equal to $\mathrm{N} \equiv \mathrm{N}_{*}=M\left(a_{*} / a_{0}\right)^{3}$, where $a_{0}$ is a characteristic size of an atomic cluster (crystal structure seed), $M$ is a number of atoms in cluster. From (25) we obtain that the law of growth looks like:

$$
\langle a\rangle_{s f} \cong\left(\frac{75 k_{B} T a_{*}^{3}}{8 A m_{u}}\right)^{1 / 5} t^{2 / 5} .
$$


Equation (56) determines growth rate of Pu crystals in the mode similar to a free-molecular flux of seeds. In this case nanoparticles play a role of seeds. From considerations of translation symmetry we will accept, for example, that the minimum size of $\delta \mathrm{Pu}$ initial nanoparticle is equal to $a_{*}=2 a_{c}=0.9274 \mathrm{~nm}$. Then with $T=700$ $\mathrm{K}, t=10$ days we obtain that $\langle a\rangle=10 \mathrm{~mm}$. The estimate corresponds in order of magnitude to the experimental data given in [67] on Pu large crystal growth by the "strain-annealing" method. Formula (56) can be useful when choosing conditions for growing large crystals of any material with strong interatomic bonds by the method of nanopowder sintering.

\section{Discussion of Results}

The above obtained approximate analytical expressions for characteristics of object formation include fundamental physical constants, physical properties of substances, and phenomenological parameters of objects. We will consider an issue about relation (32) between time-space scales and mass scales in subatomic physics. Formally relation (32) is exactly fulfilled for Planck values of mass $m_{P l}$, length $l_{P l}=\hbar /\left(m_{P I} c\right)$ and time $t_{P l}=l_{P l} / c$ which proves validity of this relation. Using this relation it was shown in Section 3 that in quark-gluon substance least time unit corresponding to the minimum value of the length unit $a_{\text {unit }}^{\min } \sim 10^{-18} \mathrm{~m}$ that can be considered as the upper estimate of a current quark size with $m_{\text {unit }} \sim m_{u}^{c q}=5 \mathrm{MeV}$, is $t_{u n i t}^{\min } \sim 10^{-29} \mathrm{~s}$. A time unit for current quarks in the state of asymptotic freedom with a spatial scale $a_{\text {unit }}^{a f} \sim 10^{-16} \mathrm{~m}$ is $t_{\text {unit }}^{a f} \sim 10^{-26} \mathrm{~s}$. Based on relation (32) one can obtain the following estimate for the unit time in processes of interaction with participation of constituent quarks in the state of confinement: $a_{\text {unit }}^{\text {conf }} \sim 10^{-15} \mathrm{~m}$, $m_{\text {unit }}=m_{u} \cong m_{d} \cong 300 \mathrm{MэB} \Rightarrow t_{\text {unit }}^{\text {conf }} \sim 4 \times 10^{-24} \mathrm{~s}$. Time of transition from asymptotic freedom to confinement is evaluated in Section 3 as $t_{\text {trans }} \sim 10^{-23} \mathrm{~s}$ that corresponds to the time scale of strong interaction, and the time of stable hadron formation is evaluated as $t_{\text {form }}^{\text {hadr }} \sim 10^{-20} \mathrm{~s}$. Thus the relation (32) in principle shows that processes in microcosm are characterized by a spectrum of time units of "measurement". It should be noted that close to the upper boundary this spectrum is overlapped with the "lower" region of the spectrum of typical nuclear times $10^{-23}-10^{-22} \mathrm{~s}$ in case of direct reactions. In this way Formula (32), demonstrating that up to distances of about $10^{-18}-10^{-16} \mathrm{~m}$ and times of about $10^{-29}-10^{-26} \mathrm{~s}$ the "ordinary" space-time relations are valid, is in compliance with generally accepted concepts about space-time scales in microcosm [68].

One can try to estimate the value of fundamental mass $m_{\text {fund }}$, if one accepts that the least space unit (fundamental length) is the value $a_{\text {fund }} \sim 10^{-18} \mathrm{~m}$, and that this value is in compliance with the time scale $t_{u n}=a_{\text {fund }} / \mathrm{c}$. Then from relation (32) we obtain the following expression for fundamental mass:

$$
m_{\text {fund }}=\frac{\hbar}{c a_{\text {fund }}} .
$$

Hence we obtain that $m_{\text {fund }} c^{2}=\hbar c / a_{\text {fund }}=196 \mathrm{GeV}$ [25]. This value fits into the expected (indicated in book [29]) experimental range in which neutral Higgs $H^{0}$-boson mass $m_{H^{0}}$ should fall in: $115<m_{H^{0}} c^{2}<250 \mathrm{GeV}$. In addition this value is close to the "critical" mass $180-200 \mathrm{GeV}$ above which $H^{0}$-boson can disintegrate into couples of W- and Z-bosons [69].The reason for this compliance is in the fact that the proposed model of "quantum" compact cluster formation is scalar, it does not include spin parameters or object charges, and hence it can be applied to the scalar neutral boson $H^{0}$ with zero spin.

It should be interesting to perform, using relation (32), a qualitative analysis of a wide spectrum of processes with the participation of fundamental and elementary particles. For example, one can try to estimate the state of the heaviest of available quarks: $t$-quark ( $t q$ ) having conditional mass $176 \mathrm{GeV}$ [19]. Using Formula (32) the minimum time unit for $t$-quark, corresponding to the minimum length unit estimated in [40] as $a_{\text {unit }}^{\text {min }} \sim 10^{-18} \mathrm{~m}$, is evaluated as $t_{\text {unit }}^{\text {tq }} \sim 10^{-27} \mathrm{~s}$. Then it follows from Formula (21) that for formation of a bound state with a characteristic size of about $10^{-15} \mathrm{~m}$, the required time would be $t_{\text {trans }} \sim 10^{-21} \mathrm{~s}$. The resultant estimate corresponds to the lifetime of unstable resonances; therefore $t$-quark cannot form stable hadrons. The result does not contradict to the known concepts according to which $t$-quark is the only quark that comes into being and dies free [19].

Within the nuclear scales the proposed method allows one to estimate the time of the process of approaching equilibrium based on characteristic mass numbers and the corresponding nuclide sizes. Such task was solved in [70] to fit the study into kinetics of deep inelastic interaction between a beam of heavy copper ions and a target of gold with the collision energy $365 \mathrm{MeV}$ [39]. It has been demonstrated that the times of formation of interac- 
tion products, having average mass numbers $\langle A\rangle=60$ and 100 typical for the process under consideration, are in the order of magnitude $10^{-20} \mathrm{~s}$. The resultant values are significantly lower than the lifetime $10^{-16}-10^{-14} \mathrm{~s}$ of an intermediate compound nucleus. This allows us to speak about relatively fast (explosive) process progress. It should be noted that the established in [39] product distribution behavior in the reaction corresponds qualitatively to asymptotic properties of a distribution density function established in Sections 2 and 3. Thus for large mass numbers law (4) is approximately fulfilled, and against the background of a smooth curve local maximums take place at $A=56$ and 63. Calculations based on Formula (28) show that the most probable mass numbers of cluster nuclides in the interregion between cluster radioactivity and spontaneous fission are equal to 51, 58, 65, 67.

In case of mesostructures the developed qualitative models include the constants $\hbar, m_{u}, k_{B}$, physical characteristics of the material $\vartheta_{V}, \theta_{D}, A, n, a_{c}, N_{c}$, phenomenological parameters $a_{0}, l_{\text {cov }}$, and operate with the minimum necessary number of variable in the experiment parameters (observed values): the environment temperature $T$, the grain initial size $l$, the time $t$ of object growth process and their average size $\langle a\rangle$ reached by the end of the process. It should be noted that the constant $\hbar$ is omitted from final expressions for an average size of volume-packed mesoobjects. This factor is connected with a large number of elementary acts of object interactions, i.e., with quasi-classical nature of growth processes of nanostructured diamond particles and light actinides. And yet, the expressions for a typical diameter and an average length of a single-wall carbon nanotube include constant $\hbar$ explicitly. This makes it possible to consider carbon nanotube as a purely quantum object.

The proposed in Ref. [15] [53] mechanism of macroscopic diamond particle formation from nanodiamonds covered all the available at that time data related to both artificial diamonds produced under static and dynamic synthesis, and natural diamonds that could have been formed as a result of cataclysm, e.g., impact of meteorite. In addition we will consider an issue about mesoscopic "limits" based on Formula (26) for the maximum size of a crystal cluster. As $\Delta t_{\min }$ it is reasonable to take the residence time of seed at one place on the surface of a particle-cluster, i.e., characteristic oscillations period $2 \pi \hbar / k_{B} \theta_{D}$. One can see that the above limits significantly depend on the seed size. By the example of diamond we will consider the following types of seeds: carbon skeleton of cyclohexane with the characteristic size $0.25 \mathrm{~nm}$, as well as nanodiamonds with the "critical" size $a_{*}=2 \mathrm{~nm}$ at which quasi-long range crystal ordering originates (see Section 4). For diamond the value $\Delta t_{\min }$ is $2.12 \times 10^{-14} \mathrm{~s}$. If we consider synthesis from molecules of carbon skeleton of cyclohexane, then the maximum size turns out to be $a_{\max }=100 \mathrm{~nm}$. This value can be considered as the "theoretical" limit of detonation nanodiamonds. If a seed size is equal to $2 \mathrm{~nm}$, then the maximum size at $\Delta t_{\min }=2.12 \times 10^{-14} \mathrm{~s}$ is evaluated as $a_{\max } \approx$ $3 \mathrm{~cm}$.

Like a theory of fuzzy martensitic transitions developing according to diffusionless mechanism [10], the model [15] [60] of the $\delta$-Pu $\rightarrow \alpha$-Pu transformation, as diffusion formation of crystal particles, shows that with the decreased grain size $l$ of the initial phase, the temperature $T_{0}$ of the martensitic transition beginning decreases, and with $l<l_{\min }$ martensitic transition is "blocked". Yet, as $l$ decreases in diffusion model the temperature $T_{*}$ of martensitic transition finish increases and "fuzzing of temperature transformation" decreases, which is determined in [25] as the difference $T_{0}-T_{*}$. It is connected with phonon effect (in particular, "zero" crystal lattice oscillations). The established in section 4 agreement between the calculated and experimental data for plutonium provides grounds for using the model in describing behavior of other light actinides with strong approach of atoms [9] (Pa, U, Np). In addition the model [15] [60] predicts the possible reverse $\alpha$-Pu $\rightarrow \delta$-Pu phase transformation under pulsed heating of the sample preliminary cooled down to temperatures of about $100 \mathrm{~K}$ and the absence of the reverse pulsed transformation in the sample preliminary cooled down to temperatures of about $15 \mathrm{~K}$.

With all variety of above processes one can single out the following common features of growth dynamics of compact objects having strong bonds and significantly different space scales:

- At the end of the aggregation process the function of distribution density $\varphi(a, t)$ of large clusters with sizes $a \gg a_{0} \quad$ ( $a_{0}$ is seed size) is in inverse proportion to $a^{3}$ (to cluster masses), and an average size $\langle a\rangle$ of clusters is much lower than the maximum size $a_{\max }:\langle a\rangle \ll a_{\max }$;

- There are many local maximums of function $\varphi(a, t)$ connected with the most probable cluster sizes, and there is a set of zeros of this function reflecting cluster instability at certain sizes;

- The obtained approximate laws of average size growth of large clusters in time $\langle a\rangle \propto t^{z}$ with small and large seed fluxes describe aggregation processes both in microcosm and in mesoscopic scales.

Every power exponent $Z$ with $t$ obtained in this work are positive which is indicative of suppression of an inverse damage wave and formation of resultant direct wave of object growth even in case of cluster interaction 
between themselves. This result is in agreement with the concept of "persistence" in cluster-cluster aggregation [8]. By analogy with the theory of dispersive waves [71] one can try to introduce the concept of "phase" and "group" velocities in dimensionless space $\xi=a / a_{0}$. Phase velocity of growth process is infinitely large: $\mathrm{d} \xi / \mathrm{d} \tau \rightarrow \infty$. This means extremely fast spreading of any initial cluster distribution and "instant" formation of density wave $\varphi(\xi, \tau)$ in space $\xi$. Group velocity $\Psi=\mathrm{d}\langle\xi\rangle / \mathrm{d} \tau$ of density wave in the majority of growth mechanisms decreases with time:

$$
\mathrm{d}\langle\xi\rangle / \mathrm{d} \tau \propto \tau^{\mathrm{Z}-1}, \quad 0<\mathrm{Z} \leq 1
$$

This reflects broadening of wave packet and slowing down of its propagation. At the final stage of the process of irreversible cluster aggregation in closed system such wave packet of a non-Gaussian shape has a decaying sudden change in a wave "front", associated with the maximum possible size $\xi_{\max }$ :

$$
\begin{gathered}
\varphi \propto \xi^{-3},\langle\xi\rangle \ll \xi \leq \xi_{\max } ; \\
\varphi=0, \xi>\xi_{\max }
\end{gathered}
$$

These considerations bring to a conclusion that the developed asymptotic method for investigation into kinetics of formation of compact objects having strong internal bonds has a certain degree of generality to use it in solving problems in physics of high density energy and high-intensive processes.

In particular, in [25] the consideration is given to a problem of nuclide "stability islands" [29] [43], and the fact of such islands existence is explained in terms of formation of clusters with the most probable sizes from nuclear matter seeds. As an example the element roentgenium with $Z=111$ has the mass number of $A=273$. In the proposed model, this value of $A$ obtained from Formula (28) corresponds to tritons as the seeds. At $Z=114$, in compliance with $2 \beta$-stability, the number of neutrons is equal to 184 and the mass number is equal to $A=298$ [43]. In the model [25], the mass numbers closed to it are 299 and 302. These values obtained from the Formula (28) correspond to tritons and deuterons as the seeds, respectively. Work [72], which is dedicated to the production and the decay of element 114, mentions its isotopes with $A=288$ and 289. In the model [25], the values $A=$ 290 and 293 are the closest mass numbers corresponded to the tritons and deuterons as the seeds, respectively. Using various calculation options of the $\beta$-stability band the islands of stability are predicted also at $Z=164$ and with neutron numbers of 272 or 318 [43]. Respective mass numbers $A=436$ and 482 are close to the approximate value of the mass number of the final nuclide $A_{\text {end }} \approx 470$ calculated in [14]. In the model [25], mass number $A=436$ corresponds to deuterons as the seeds. The closest to the mass number of 482 are the following values calculated with the help of Formula (28): $A=483$ for the tritons as the seeds, and $A=485$ for deuterons as the seeds. Thus, one can presume that the developed asymptotic model of clusters formation in the nuclear matter complements the method for predicting nuclear mass with the help of radial basis function [73], which makes it possible to find mass numbers in the range from 20 to 260.

As for high-intensive nuclear processes, when a target is affected by fast, including relativistic, particles, then to describe them one should use Equation (25). From this equation as well as from Formula (27) one can derive the following expression for the time of formation of cluster nuclides with an average mass number $\langle A\rangle$ :

$$
t_{\text {form }} \cong 2 r_{0}\left(\frac{2 m_{\text {nucl }}}{E_{\text {exc }}}\right)^{1 / 2}\langle A\rangle^{1 / 3}
$$

Here, $m_{\text {nucl }}$ is nucleon mass, which can be accepted as an average value between proton and neutron masses, $E_{\text {exc }}$ is nucleus excitation energy per a nucleon. If we accept the average kinetic nucleon energy in degenerate ideal Fermi-gas as $E_{\text {exc }}$, then from the previous equation we will have:

$$
t_{\text {form }}^{a c} \cong \frac{4 r_{0}}{c_{0}}\langle A\rangle^{1 / 3}
$$

Here, $c_{0}$ is average thermal nucleon velocity (see Section 3). In such "acoustic" (ac) approximation the time of light nuclide formation with $\left\langle A>\approx 10\right.$ is equal to $2 \times 10^{-22} \mathrm{~s}$, for heavy nuclides with $<A>\approx 100$ this time is 5 $\times 10^{-22} \mathrm{~s}$. If one takes the maximum possible energy $m_{\text {nucl }} c^{2}$ as $E_{\text {exc }}$, the time of nuclide cluster formation is expressed as:

$$
t_{\text {form }}^{u r} \cong \frac{2 \sqrt{2} r_{0}}{c}\langle A\rangle^{1 / 3}
$$


In such "ultrarelativistic" (ur) approximation the times of nuclide formation are $2 \times 10^{-23} \mathrm{~s}$ and $4 \times 10^{-23} \mathrm{~s}$. The obtained estimates do not contradict to the generally known notions about typical time of direct nuclear reactions [29] [35] [36].

Another example of using the developed method is explanation and description of diamond synthesis in conditions of cavitation experimentally studied in [74] [75]. In [76] a solid stage of the process is considered, at this stage diamond particle growth in extreme condition occurs. Asymptotic models of nanostructured mesoobjects with strong interatomic bonds make the basis of this consideration [15]. These models include description of crystal growth models at high temperatures $T$, when $k_{B} T \gg \hbar \omega$. For obtaining laws of diamond particle growth in conditions of cavitation Equation (25) was used. It has been shown that nanodiamonds are produced at the first stage of synthesis. Hence production of diamond particles in a solid phase occurs as a result of nanodiamond coalescence and consolidation of the produced objects into larger particles both at the final stage of cavitation bubble collapse, and in the process of collapse "products" expansion as well.

The next example is the problem of determining the width of detonation zone in solid high explosives (HE) [77]. In this case the subject to be investigated is not formation of an object with strong internal bonds, but failure of strong covalent bonds between carbon atoms, resulting in relaxation processes of new bonds formation and energy release. The detonation zone is considered as quantum mechanical system, being in an excited state. For example, it can be excitation of quasi-particles, phonons, caused by shock wave. As a result of system phonon excitations, there occur atom oscillations with relatively high amplitude with which bonds between atoms break and new bonds are formed that are typical for decomposition products of the original substance: various chemical compounds (molecules, radicals), carbon clusters, and others.

Relaxation processes in a detonation are considered using the concept of distribution density wave $\varphi(x, t)$ of objects with new interatomic bonds, formed when former bonds broke as a result of "excitation" of oscillations. The wave $\varphi(x, y)$ propagates with time $t$ within a quantum mechanical system along the axis of HE charge ( $x$-axis) from the shock wave front towards the surface, where chemical reactions of energy release finish. The motion of the wave $\varphi$ within the detonation zone goes up to the time $t=\tau$, where $\tau$ is duration time of detonation-related chemical reactions. The estimates obtained for the average (in terms of its charge width section) detonation zone fit into the range of generally known data for typical solid HE, and indicate that it is possible to produce explosives with nanometer energy release regions [77].

One can try to apply the proposed asymptotic method to estimate grain sizes formed as a result of recrystallization process in aluminum alloys at an impact of a group of solids with a rate of $1200-1300 \mathrm{~m} / \mathrm{s}$ [78]. Numerical calculations have shown that the material temperature of crater walls formed, when solid impactors penetrate aluminum alloy targets, is $T=500 \mathrm{~K}$, and a full time of impactor penetration up to its stopping is $t=40 \mu \mathrm{s}$. One can accept this calculated time as an estimate of life duration of intensive phonon excitations in the system "impactor-target material”. As in a previous example, the process of grain formation and growth can be represented as a process of coalescence and growth of nanocrystals formed under failure of target original material structure as a result of solid shock action and stress wave interference generated by neighboring impactors.

Since the calculated temperature is comparable with Debye aluminum parameter $\theta_{D}=443 \mathrm{~K}$ [79], then with small flux of nanocrystal seeds the time dependence of an average size of crystal particles (grains) is described by Formula (45). From here, we get that at $t=40 \mu \mathrm{s}$, the grain average size is $0.4 \mu \mathrm{m}$. This value can be accepted as a grain size estimate at primary recrystallization. This estimate corresponds to grain sizes at a crater bottom specified in [78] using scanning electronic microscope.

In general, the proposed phenomenological approach seems to be useful when considering the following problems from the list given in review [68]: mesoscopics, clusters, nanotubes, super-heavy elements, mass spectrum, quarks and gluons, fundamental length, particle interaction at high energies, neutron stars, and supernovas.

It should be noted that the estimated in [25] fundamental mass $196 \mathrm{GeV}$ does not differ strongly from the mass 125 - $126 \mathrm{GeV}$ [80] of the new particle with Higgs boson properties discovered on the Big Hadron collider in CERN. It could not be ruled out that the above new particle is a product of disintegration of another heavier particle. It is also important to mark approximate accordance of obtained evaluation for $m_{\text {fund }}$ to the value of the upper limit of the Higgs mass identified in [81] as $170 \mathrm{GeV}$. These factors argue in favor of validity of relation (32) in terms of fundamental processes in microcosm.

Hence the developed models can be useful both for theoretical studies and experiments as well as for primary analysis of their first data. 


\section{Conclusions}

The proposed phenomenological approach allows one to consider formation and growth of subatomic and mesoscopic objects under single formalism.

Based on linearization and qualitative analysis of solutions of Fokker-Planck kinetic equation the basic properties of a size-distribution density function of volume-packed bodies were established and approximate relations between average object sizes and their growth times were obtained.

An approximate differential relation for determining object growth in high-intensive processes with high energy was obtained.

The proposed asymptotic model provides adequate estimates of mass characteristics of typical intranuclear processes.

The calculated data obtained for formation of nanostructured mesoobjects having strong interatomic bonds agree well with generally known experimental data.

\section{References}

[1] Morokhov, I.D., Petinov, V.P., Turusov, L.P. and Petrunin, V.F. (1981) Structure and Properties of Fine Metallic Particles. Soviet Physics Uspekhi, 24, 295-334. http://dx.doi.org/10.1070/PU1981v024n04ABEH004800

[2] Kadomtsev, B.B. (1994) Dynamics and Information. Soviet Physics Uspekhi, 37, 425-499. http://dx.doi.org/10.1070/PU1994v037n05ABEH000109

[3] Von Oppen, G. (1994) Objects and Environment. Physics-Uspekhi, 39, 617-620. http://dx.doi.org/10.1070/PU1996v039n06ABEH000152

[4] Smirnov, B.M. (2011) Processes Involving Clusters and Small Particles in Buffer Gas. Physics-Uspekhi, 54, $691-721$.

[5] Pool, C.P. and Owens, F.J. (2003) Introduction to Nanotechnology. John Wiley \& Sons Inc., London.

[6] Imry, Y. (2002) Introduction to Mesoscopic Physics. Oxford University Press, Oxford.

[7] Bording, J.K. and Taft, J. (2000) Molecular-Dynamics Simulation of Growth of Nanocrystals in Amorphous Matrix. Physical Review B, 62, 8098-8103. http://dx.doi.org/10.1103/PhysRevB.62.8098

[8] Hellen, E.K. and Alava, M.J. (2002) Persistence in Cluster-Cluster Aggregation. Physical Review E, 66, 026120.

[9] Hecker, S.S. (2000) Plutonium and Its Alloys: From Atoms to Microstructures. In: Cooper, N.G., Buican, I.G. and Schecker, J.A., Eds., Challenges in Plutonium Science, Los Alamos Science, Los Alamos, 290-334.

[10] Malygin, G.A. (2008) Nanoscope Size Effects on Martensitic Transformation in Shape Memory Alloys. Physics of the Solid State, 50, 1538-1543. http://dx.doi.org/10.1134/S1063783408080258

[11] Lin, E.E. (1993) Kinetics of the Formation of Compact Quantum Clusters in Stochastic Conservative Systems. Technical Physics Letters, 19, 165-166.

[12] Suzdalev, I.P. (2006) Nanotechnology: Physics-Chemistry of Nanoparticles, Nanostructures and Nanomaterials. Synergetics: From the Past to Future, Komkniga, Moscow. (in Russian)

[13] Vanossi, A., Manini, N., Urbakh, M., Zapetti, S. and Tosatti, E. (2013) Modeling Friction: From Nanoscale to Mesoscale. Review of Modern Physics, 85, 529-562. http://dx.doi.org/10.1103/RevModPhys.85.529

[14] Lin, E.E. (2012) Calculating the Mass Numbers of Cluster Nuclides on the Basis of the Asymptotic Model. Bulletin of the Russian Academy of Sciences: Physics, 76, 881-883. http://dx.doi.org/10.3103/S1062873812080199

[15] Lin, E.E. (2010) Mesokinetics of Growth of Nanostructured Objects with Strong Interatomic Bonds. In: Taylor, J., Ed., Advances in Chemistry Research, Nova Science Publishers, New York, 5, 171-190.

[16] Fermi, E. (1960) Notes on Quantum Mechanics. The University of Chicago Press, Chicago.

[17] Man’ko, V.I. (1990) Coherent State. In: Prokhorov, A.M., Ed., Physical Encyclopedia, Soviet Encyclopedia, Moscow, 392-394. (in Russian)

[18] Wagner, P. and Zhong, Y.M. (1995) Cluster Formation in Disordered Systems and Nuclear Fragmentation. Nuclear Physics A, 592, 385-412.

[19] Okun, L.B. (1998) Current Status of Elementary Particle Physics. Physics-Uspekhi, 41, 553-557. http://dx.doi.org/10.1070/PU1998v041n06ABEH000403

[20] Dremin, I.M. (2010) The Quark-Gluon Medium. Physics-Uspekhi, 53, 123-1149.

[21] Lozovik, Y.E. and Popov, A.M. (1997) Formation and Growth of Carbon Nanostructures: Fullerens, Nanoparticles, Nanotubes and Cones. Physics Uspekhi, 40, 717-737. http://dx.doi.org/10.1070/PU1997v040n07ABEH000253

[22] Mukhin, K.N. and Patarakin, O.O. (2000) Exotic Processes in Nuclear Physics. Physics-Uspekhi, 43, 799-839. 
http://dx.doi.org/10.1070/PU2000v043n08ABEH000802

[23] Lin, E.E. (2010) Asymptotical Model of the Formation of Nuclear Matter Clusters. In: Il'kaev, R.I., Ed., Proceedings of International Conference 12th Khariton Thematic Scientific Readings, Problems of High Density Energy Physics, 19-23 April 2010, Sarov, 262-267. (in Russian)

[24] Panov, I.V. and Thielemann, F.K. (2004) Fission and the $r$-Process: Competition between Neutron-Induced and BetaDelayed Fission. Astronomic Letters, 30, 647-655. http://dx.doi.org/10.1134/1.1795953

[25] Lin, E.E. (2011) Qualitative Kinetic Models of Formation of Compact Objects with Strong Internal Bonds. Monograph, Russian Federal Nuclear Center-VNIIEF, Sarov. (in Russian)

[26] Pugachev, V.C. and Sinitsyn, I.N. (1990) Stochastic Differential Systems: Analysis and Filtration. Nauka, Moscow. (in Russian)

[27] Reissland, J.A. (1973) The Physics of Phonons. John Wiley and Sons LTD., London; New York; Sydney; Toronto.

[28] Kulakov, V.M. (1991) Nuclear Properties of Nuclides. In: Grigor'ev, S. and Meilikhov, E.Z., Eds., Physical Quantities Handbook, Energoatomizdat, Moscow, 993-1054. (in Russian)

[29] Ishanov, B.S., Kapitonov, I.M. and Yudin, N.P. (2007) Particles and Atomic Nuclei. LKI, Moscow. (in Russian)

[30] Kadmenskii, S.G., Kurgalin, S.D. and Tchuvil'sky, Y.M. (2012) Cluster States in Atomic Nuclei and Cluser-Decay Processes. Physics of Particles and Nuclei, 38, 699-742.

[31] Zamyatnin, Y.S., Kadmensky, S.S., Kurgalin, S.D. and Tchuvil’sky, Y.M. (1994) Where the New Examples of Nuclei Cluster Decay Can Be Seek? Physics of Atomic Nuclei, 57, 1905-1918.

[32] Vandenbosch, R. and Huisenga, J.R. (1973) Nuclear Fission. Academic Press, New York; London.

[33] Obukhov, A.I. and Grigor'ev, I.S. (1991) Fission of Nuclei. In: Grigor'ev, I.S. and Meilikhov, E.Z., Eds., Physical Quantities Handbook, Energoatomizdat, Moscow, 1087-1098. (in Russian)

[34] Panov, I.V., Korneev, I.Y. and Thielemann, F.K. (2009) Superheavy Elements and r-Process. Physics of Atomic Nuclei, 72, 1026-1033. http://dx.doi.org/10.1134/S1063778809060155

[35] Olkhovsky, V.S. (1984) On Investigation of Nuclear Reactions and Decays with the Help of Analysis of Theirs Durations. Soviet Journal of Particles and Nuclei, 15, 130-148.

[36] Olkhovsky, V.S., Recami, E. and Maydanuyk, S.P. (2012) Time as Quantum Observable, Canonical Conjugated to Energy. In: Pahlavani, M.R., Ed., Measurements in Quantum Mechanics, In Tech, Shaghai, 18-56.

[37] Segre, E. (1964) Nuclei and Particles, an Introduction to Nuclear and Subnuclear Physics. W. A. Benjamin Inc., New York.

[38] Segre, E. (1977) Nuclei and Particles. 2nd Edition, W. A. Benjamin Inc., New York.

[39] Valentin, L. (1982) Physique Subatomique: Noyaux Et Particles. Nouvelle Edition Entirement Refondue, Hermann, Paris.

[40] Jacob, M. and Landshoff, P. (1982) The Inner Structure of Proton. Scientific American, 242, 66-75. http://dx.doi.org/10.1038/scientificamerican0380-66

[41] Kane, G. (1987) Modern Elementary Particle Physics. University of Michigan, Addison-Wesley Publishing Company Inc., Michigan.

[42] Neufeld, R.B. and Mueller, B. (2009) Sound Produced by a Fast Parton in a Guark-Gluon Plasma Is a "Crescendo”. Physical Review Letters, 103, 043201.

[43] Selinov, I.P. (1990) Atomic Nuclei: Structure and Systematic. Nauka, Moscow. (in Russian)

[44] Nadezin, D.K. (1992) Neutron Stars. In: Prokhorov, A.M., Ed., Physical Encyclopedia, Big Russian Encyclopedia, Moscow, 280-283. (in Russian)

[45] Utrobin, V.P. (1994) Supernovae. In: Prokhorov, A.M., Ed., Physical Encyclopedia, Big Russian Encyclopedia, Moscow, Vol. 4, 433-435. (in Russian)

[46] Ponomarev, L.I. (1992) Uncertainty Relations. In: Prokhorov, A.M., Ed., Physical Encyclopedia, Big Russian Encyclopedia, Moscow, 321-322. (in Russian)

[47] Vereshchagin, A.L., Sakovich, G.V., Brylyakov, P.M., Zolotukhina, I.I., Petrova, L.A. and Novoselov, N.N. (1990) The Structure of Carbon Diamond-Like Phase of Detonated Synthesis. Soviet Physics Doklady, 35, 851-852.

[48] Lin, E.E. (2003) On the Growth Kinetics of a Single-Wall Carbon Nanotube. Doklady Physics, 48, $180-181$. http://dx.doi.org/10.1134/1.1574375

[49] Badiali, J.P. (1999) Fractal Behavior in Quantum Statistical Physics. Physical Review E, 60, 2533-2539. http://dx.doi.org/10.1103/PhysRevE.60.2533

[50] Vollmer, M. (1983) Kinetics of New Phase Formation. Plenum, New York. (Kinetic der Phasenbildung. T. Steinkopf, 
Dresden, 1939)

[51] Syue-sen', T. (1965) Physical Mechanics. Mir, Moscow. (Translated from Chinese)

[52] Yakubov, T.S. (1990) On Specific Heat of Solids Revealing Fractal Character. Doklady Academii Nauk SSSR, 310, 145-149.

[53] Lin, E.E. (2000) On the Cluster Mechanism of Diamond Synthesis from Different Solid Carbon Forms. Physics of the Solid State, 42, 1946-1951. http://dx.doi.org/10.1134/1.1318892

[54] Lin, E.E. (1994) Aggregation of Crystalline Clusters in the Shock Wave Front Propagating through Condensed Species. Soviet Journal of Chemical Physics, 12, 404-409.

[55] Lin, E.E. (1997) Shock-Induced Growth of Crystals in a Porous Medium of Diamond Nanoparticles. Chemical Physics Reports, 16, 2241-2244.

[56] Lin, E.E. (2000) Shock-Induced Coalescence of Nanodiamonds. Bulletin of the Russian Academy of Sciences: Physics, 64, $1215-1216$.

[57] Lin, E.E. (2005) Pulsed Loading of Objects during Intense Expansion of Products of Solid Explosives (Review). Combustion, Explosion, and Shock Waves, 41, 241-263. http://dx.doi.org/10.1007/s10573-005-0030-2

[58] Lin, E.E. (2009) Determining the Growth Rate of Nanostructured Particles of Light Actinide at High and Moderate Temperatures. Technical Physics Letters, 35, 418-420. http://dx.doi.org/10.1134/S1063785009050101

[59] Van Bueren, H.G. (1960) Imperfections in Crystals. North-Holland Publishing Company, Amsterdam.

[60] Lin, E.E. (2010) Influence of Size Factors on the Character of Size Transformations in Light Actinides. Physics of the Solid State, 52, 153-157. http://dx.doi.org/10.1134/S1063783410010270

[61] Janke, E., Emde, F. and Losch, F. (1960) Tafeln Hoherer Funktionen. (Neubearbeitet von F. Losch), B. G. Verlagsgesellschaft, Stuttgart.

[62] Louson, E.S., Martinez, B., Roberts, J.A., Richardson, J.U. and Benne, B.I. (2000) Oscillations of Atoms and Plutonium Melting. In: Cooper, N.G., Buican, I.G. and Schecker, J.A., Eds., Challenges in Plutonium Science, Los Alamos Science, Los Alamos, 190-199.

[63] Dwight, H.B. (1961) Tables of Integrals and other Mathematical Data. The Macmillan Company, New York.

[64] Hecker, S.S., Harbur, D.R. and Zocco, T.G. (2004) Phase Stability and Phase Transformations in Pu-Ga Alloys. Progress in Materials Science, 49, 429-485. http://dx.doi.org/10.1016/S0079-6425(03)00032-X

[65] Kitching, S., Planterose, P.G. and Gill, D.C. (2003) Stabilized Plutonium. In: Jarvinen, G.D., Ed., Plutonium FuturesThe Science, American Institute of Physics, New York, 79-81.

[66] Mitchell, J.N., Hecker, S.S., Freibert, F.J., Schwarts, D.S. and Bange, M.E. (2008) Inconventional Delta-Phase Stabilization in Pu-Ga Alloys. In: Fundamental Plutonium Properties, Proceedings of 8th International Workshop, 8-12 September 2008, Snezhinsk, 5-6.

[67] Leshli, D.S., Blau, M.S. and Moment, R.L. (2000) Manufacturing of Single Crystals of Plutonium. In: Cooper, N.G., Buican, I.G. and Schecker, J.A., Eds., Challenges in Plutonium Science, Los Alamos Science, Los Alamos, 233-245.

[68] Ginzburg, V.L. (1999) What Problems of Physics and Astrophysics Seem Now to Be Especially Important and Interesting (Thirty Years Later, Already on the Verge of XXI Century)? Physics-Uspekhi, 42, 353-373. http://dx.doi.org/10.3367/UFNr.0169.199904d.0419

[69] Okun, L.B. (1985) Particle Physics: The Guest for the Substance of Substance. Hardwood Academic Publishers, Chur.

[70] Lin, E.E. (1993) Kinetics of Deep-Inelastic Heavy Ions Interaction. Technical Physics Letters, 19, 669-670.

[71] Whitham, G.B. (1974) Linear and Nonlinear Waves. A Wiley-Interscience Publication, Hoboken.

[72] Düllmann, C.E., Schädell, M. and Yakushev, A. (2010) Production and Decay of Element 114: High Cross Sections and New Nucleus ${ }^{277}$ Hs. Physical Review Letters, 104, 252701-252705. http://dx.doi.org/10.1103/PhysRevLett.104.252701

[73] Wang, N. and Liu, M. (2011) Nuclear Mass Predictions with a Radial Basis Function Approach. Physical Review C, 84, 051303.

[74] Galimov, E.M., Kudin, A.M., Skorobogatskii, V.N., Plotnichenko, V.G., Bondarev, O.L., Zarubin, B.G., Strazdovskii, V.V., Aronin, A.S., Fisenko, A.V. and Bubov, I.V. (2004) Experimental Confirmation of the Diamond Synthesis during Cavitation. Doklady Physics, 395, 187-191.

[75] Khachatryan, A., Aloyan, S.S., May, P.W., Sargsyan, R., Khachatryan, V.A. and Bagdasaryan, V.S. (2008) Grafite-toDiamond Transformation Induced by Ultrasound Cavitation. Diamond and Related Materials, 17, 931-936. http://dx.doi.org/10.1016/j.diamond.2008.01.112

[76] Lin, E.E. (2011) Cluster Mechanism of Diamond Synthesis under Severe Cavitation Conditions. Technical Physics Le- 
tters, 37, 593-595. http://dx.doi.org/10.1134/S1063785011070121

[77] Lin, E.E. (2011) Detonation Zone Width Determination Based on Uncertainty Principle. Technical Physics Letters, 37, 449-450. http://dx.doi.org/10.1134/S1063785011050257

[78] Auchev, A.A., Ilyushkina, N.Y., Lin, E.E., Popov, N.N. and Tanakov, Z.V. (2013) Recrystallization in Aluminum Alloyes under Impact of Solids. In: Proceedings of International Conference 15th Khariton Topical Scientific Readings “Extreme States of Substance. Detonation. Shock Waves”, 18-22 March 2013, Sarov, 300-301. (in Russian)

[79] Hlopkin, M.N. (1991) Heat Capacity. In: Grigor’ev, S. and Meilikhov, E.Z., Eds., Physical Quantities Handbook, Energoatomizdat, Moscow, 205. (in Russian)

[80] Rubakov, V.A. (2012) Large Hadron Collider’s Discovery of a New Particle with Higgs Boson Properties. PhysicsUspekhi, 55, 949-957.

[81] Elsayed, A., Khalil, S. and Moretti, S. (2012) Higgs Mass Corrections in the SUSY B-L Model with Inverse Seesaw. Physics Letters B, 715, 208-213. http://dx.doi.org/10.1016/j.physletb.2012.07.066 
Scientific Research Publishing (SCIRP) is one of the largest Open Access journal publishers. It is currently publishing more than 200 open access, online, peer-reviewed journals covering a wide range of academic disciplines. SCIRP serves the worldwide academic communities and contributes to the progress and application of science with its publication.

Other selected journals from SCIRP are listed as below. Submit your manuscript to us via either submit@scirp.org or Online Submission Portal.
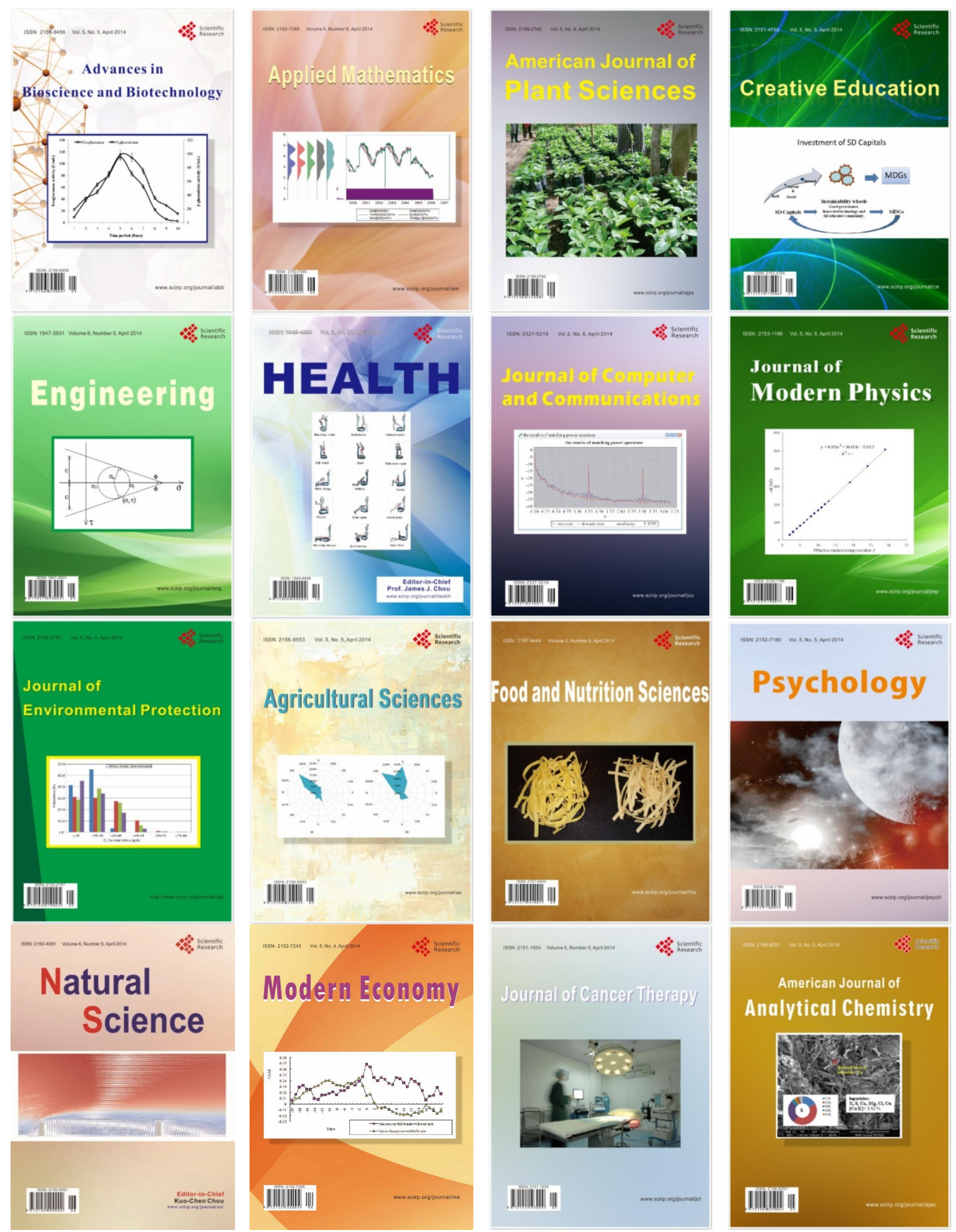